\title{
WEAK BIALGEBRAS AND MONOIDAL CATEGORIES
}

\author{
G. BÖHM, S. CAENEPEEL, AND K. JANSSEN \\ To Mia Cohen, on the occasion of her retirement.
}

\begin{abstract}
We study monoidal structures on the category of (co)modules over a weak bialgebra. Results due to Nill and Szlachányi are unified and extended to infinite algebras. We discuss the coalgebra structure on the source and target space of a weak bialgebra.
\end{abstract}

\section{INTRODUCTION}

Let $H$ be a $k$-algebra. It is well-known that bialgebra structures on $H$ are in bijective correspondence to monoidal structures on the category ${ }_{H} \mathcal{M}$ of left $H$-modules that are fibred, which means that the forgetful functor ${ }_{H} \mathcal{M} \rightarrow{ }_{k} \mathcal{M}$ is strongly monoidal.

Weak bialgebras are more general than bialgebras. The axioms $\Delta(1)=1 \otimes 1$ and $\varepsilon(h k)=\varepsilon(h) \varepsilon(k)$ are replaced by four weaker axioms (lm), (rm), (lc), (rc). Weak bialgebras and weak Hopf algebras were introduced in [3], although the history of the subject goes back to earlier notions, we refer to the introduction in [3] for more detail. For a survey on weak bialgebras (and Hopf algebras), we refer to 12 .

A natural question that arises is whether the category of representations of a weak bialgebra is monoidal. An indirect answer to this question is given by the observation that a weak bialgebra $H$ is a left bialgebroid over a separable Frobenius algebra which is denoted by $H_{t}$ in the sequel, see for example [1, Sec. 3.2]. Szlachányi [17 has reformulated the definition of a left bialgebroid in terms of monoidal categories, implying that the category of representations of a weak bialgebra is indeed monoidal and the forgetful functor to the module category of the ground ring possesses a so-called separable Frobenius structure. The product on the category of modules over a weak bialgebra is given by the tensor product over $H_{t}$. In [14, it is shown that the (co)module category of any weak bialgebra in an idempotent complete braided monoidal category is monoidal with a separable Frobenius

2010 Mathematics Subject Classification. 16T05.

Key words and phrases. weak bialgebra, monoidal category.

The first named author was supported by the Hungarian Scientific Research Fund OTKA K68195.The second and third named authors were supported by the research project G.0117.10 "Equivariant Brauer groups and Galois deformations" from FWOVlaanderen. All authors wish to thank Joost Vercruysse for fruitful discussions. 
forgetful functor. More generally, in 2] those monads (termed weak bimonads) were described whose Eilenberg-Moore category is monoidal such that the forgetful functor possesses a separable Frobenius structure.

The question about monoidality of the (co)module category of a weak bialgebra has also been investigated by Nill [13], see also [4] and [12. Actually it suffices that only two of the four axioms, namely ( $\mathrm{rm})$ and $(\mathrm{lm})$ are fulfilled, and then the representation category is monoidal. But this time the product is defined in a different way, as a subspace (or quotient) of the usual tensor product (but no longer a (co)module tensor product), and the unit object is now a subspace of the dual of the prebialgebra. Recall that Nill only looks at finite dimensional weak Hopf algebras, and this condition is needed in his result. This has the obvious advantage that the self-duality of the axioms can be fully exploited, and allows to conclude immediately that the category of comodules is monoidal once (rc) and (lc) are fulfilled.

The aim of this note is to unify and extend the above mentioned results, in a straightforward and elementary way. We look at weak bialgebras over commutative rings that are not necessary finite. Then we can show that conditions (lm) and ( $\mathrm{rm}$ ) imply that the representation category is monoidal, while (lc) and (rc) imply that the corepresentation category is monoidal. Furthermore, we prove that, under these assumptions, the respective forgetful functor is both monoidal and opmonoidal. It becomes essentially strong (op)monoidal if any third one of the listed axioms holds. In this case the monoidal product in the (co)representation category is given by a module tensor product or, isomorphically, by a comodule tensor product. The forgetful functor is of the separable Frobenius type if and only if all of the four listed axioms hold.

Now let $H$ be weak bialgebra, that is, all four axioms are satisfied. Then it can be shown that the unit object of the representation category is isomorphic to $H_{t}$, the subalgebra of $H$ that we mentioned above and that was observed by Szlachányi [17] to be a separable Frobenius algebra. General properties of Frobenius algebras then show that $H_{t}$ is also a coseparable Frobenius coalgebra, and they can be used to obtain an alternative proof of the fact that the the tensor product on the representation category is actually the comodule tensor product over $H_{t}$ and is isomorphic to the tensor product over $H_{t}$. Similar results can be proved for the corepresentation category.

The paper is organized as follows. In Section 1, we revisit the elementary properties of weak bialgebras, where we indicate which axioms are needed for each property. We will not give full detail on all the proofs, but we have tried to organize our text in such a way that the missing details can be easily filled in. Sections 2 and 3 are devoted to resp. the categories of modules and of comodules. In Section 4, we fist recall some general theory on separable Frobenius algebras, and then we apply it to weak bialgebras.

We will use standard terminology and notation from classical Hopf algebra theory, see for example [11] or [8]. In particular, we use the Sweedler 
notation for comultiplications and coactions. In the Sweedler indices, we use brackets () for comultiplication and square brackets [] for coactions. If several copies of $\Delta(1)$ occur in the same formula, then we use primes to distinguish between them: we write $\Delta(1)=1_{(1)} \otimes 1_{(2)}=1_{\left(1^{\prime}\right)} \otimes 1_{\left(2^{\prime}\right)}$. For the general theory of monoidal categories, we refer to 9 .

\section{WEAK BIALGEBRAS}

Let $k$ be a commutative ring, and $H$ a $k$-module carrying the structure of $k$-algebra and $k$-coalgebra, with unit 1 , comultiplication $\Delta$ and counit $\varepsilon$, and assume that $\Delta(h k)=\Delta(h) \Delta(k)$, for all $h, k \in H$. Then we call $H$ a $k$-prebialgebra. $H$ is called finite if $H$ is finitely generated and projective as a $k$-module. If we work over a field, then this means that $H$ is finite dimensional.

A prebialgebra is called left monoidal (resp. right monoidal, left comonoidal, right comonoidal) if condition $(\mathrm{lm})$ (resp. ( $\mathrm{rm}),(\mathrm{lc}),(\mathrm{rc}))$ holds, for all $h, k, l \in H$ :

$$
\begin{aligned}
& (\operatorname{lm}) \varepsilon(h k l)=\varepsilon\left(h k_{(1)}\right) \varepsilon\left(k_{(2)} l\right) \quad ; \quad \Delta^{2}(1)=1_{(1)} \otimes 1_{(2)} 1_{\left(1^{\prime}\right)} \otimes 1_{\left(2^{\prime}\right)} \text { (lc) } \\
& (\mathrm{rm}) \varepsilon(h k l)=\varepsilon\left(h k_{(2)}\right) \varepsilon\left(k_{(1)} l\right) \quad ; \quad \Delta^{2}(1)=1_{(1)} \otimes 1_{\left(1^{\prime}\right)} 1_{(2)} \otimes 1_{\left(2^{\prime}\right)}(\mathrm{rc})
\end{aligned}
$$

A prebialgebra that is at the same time left and right (co)monoidal is called (co)monoidal, and then we say that condition (m) (resp. condition (c)) is satisfied. (l) (resp. (r)) will mean that $H$ is a the same time left (resp. right) monoidal and comonoidal. A weak bialgebra is a monoidal and comonoidal prebialgebra. This terminology agrees with the terminology in [3]. What we call a prebialgebra is called a weak bialgebra in 13 .

Let $H^{*}$ be the linear dual of $H . H^{*}$ is a $k$-algebra, with the opposite convolution as multiplication: $\left\langle h^{*} k^{*}, h\right\rangle=\left\langle h^{*}, h_{(2)}\right\rangle\left\langle k^{*}, h_{(1)}\right\rangle$. If $H$ is finite, then $H^{*}$ is a $k$-coalgebra, with comultiplication given by the formula $\Delta\left(h^{*}\right)=h_{(1)}^{*} \otimes h_{(2)}^{*}$ if and only if $\left\langle h^{*}, h k\right\rangle=\left\langle h_{(1)}^{*}, k\right\rangle\left\langle h_{(2)}^{*}, h\right\rangle$, for all $h, k \in H$. So if $H$ is finite, then the dual of prebialgebra is again a prebialgebra.

Lemma 1.1. Let $H$ be a finite prebialgebra. $H$ satisfies (lm) (resp. (rm)) if and only if $H^{*}$ satisfies (lc) (resp. (rc)).

Proof. We will prove that $H^{*}$ satisfies (lc) if $H$ satisfies $(\mathrm{lm})$. All the other implications can be proved in a similar way. $H^{*}$ satisfies (lc) if and only if

$$
\Delta^{2}(\varepsilon)=\varepsilon_{(1)} \otimes \varepsilon_{(2)} \varepsilon_{\left(1^{\prime}\right)} \otimes \varepsilon_{\left(2^{\prime}\right)} .
$$

This is equivalent to $\langle\varepsilon, h k l\rangle$ being equal to

$$
\begin{aligned}
\left\langle\varepsilon_{(1)}, l\right\rangle\left\langle\varepsilon_{(2)} \varepsilon_{\left(1^{\prime}\right)}, k\right\rangle\left\langle\varepsilon_{\left(2^{\prime}\right)}, h\right\rangle & =\left\langle\varepsilon_{(1)}, l\right\rangle\left\langle\varepsilon_{(2)}, k_{(2)}\right\rangle\left\langle\varepsilon_{\left(1^{\prime}\right)}, k_{(1)}\right\rangle\left\langle\varepsilon_{\left(2^{\prime}\right)}, h\right\rangle \\
& =\left\langle\varepsilon, k_{(2)} l\right\rangle\left\langle\varepsilon, h k_{(1)}\right\rangle,
\end{aligned}
$$

for all $h, k, l \in H$, and this is clearly satisfied if $H$ satisfies (lm). 
If $H$ is a prebialgebra, then $H$ is an $H^{*}$-bimodule, and $H^{*}$ is an $H$-bimodule:

$$
h^{*} \rightarrow h \smile k^{*}=\left\langle k^{*}, h_{(1)}\right\rangle h_{(2)}\left\langle h^{*}, h_{(3)}\right\rangle ;\left\langle h \rightarrow h^{*} \longleftarrow k, l\right\rangle=\left\langle h^{*}, k l h\right\rangle .
$$

Now consider the maps $f, f^{\prime}: H^{*} \rightarrow H ; g, g^{\prime}: H \rightarrow H^{*}$,

$$
\begin{array}{cc}
f\left(h^{*}\right)=1 \leftarrow h^{*}=\left\langle h^{*}, 1_{(1)}\right\rangle 1_{(2)} & f^{\prime}\left(h^{*}\right)=h^{*} \rightarrow 1=\left\langle h^{*}, 1_{(2)}\right\rangle 1_{(1)} \\
g(h)=h-\varepsilon=\langle\varepsilon,-h\rangle & g^{\prime}(h)=\varepsilon^{<} \leftarrow=\langle\varepsilon, h-\rangle
\end{array}
$$

If $H$ is finite, then we can identify $H$ and $H^{* *}$, and then $f^{\prime}=f^{*}$ and $g^{\prime}=g^{*}$. If we specify in our notation that these maps depend on the prebialgebra $H$, that is, we write $f=f_{H}, g=g_{H}$, etc., then $f_{H^{*}}=g_{H}$ and $f_{H^{*}}^{\prime}=g_{H}^{\prime}$ if $H$ is finite. Now we define eight more maps $\varepsilon_{t}, \varepsilon_{s}, \bar{\varepsilon}_{t}, \bar{\varepsilon}_{s}: H \rightarrow H$ and $\varepsilon_{t}^{*}, \varepsilon_{s}^{*}, \bar{\varepsilon}_{t}^{*}, \bar{\varepsilon}_{s}^{*}: H^{*} \rightarrow H^{*}$.

$$
\begin{array}{llll}
\varepsilon_{t}=f \circ g & \varepsilon_{s}=f^{\prime} \circ g^{\prime} & \bar{\varepsilon}_{t}=f \circ g^{\prime} & \bar{\varepsilon}_{s}=f^{\prime} \circ g \\
\varepsilon_{t}^{*}=g \circ f & \varepsilon_{s}^{*}=g^{\prime} \circ f^{\prime} & \bar{\varepsilon}_{t}^{*}=g \circ f^{\prime} & \bar{\varepsilon}_{s}^{*}=g^{\prime} \circ f
\end{array}
$$

We have that $\left(\varepsilon_{t}\right)^{*}=\varepsilon_{s}^{*},\left(\varepsilon_{s}\right)^{*}=\varepsilon_{t}^{*},\left(\bar{\varepsilon}_{t}\right)^{*}=\bar{\varepsilon}_{t}^{*}$ and $\left(\bar{\varepsilon}_{s}\right)^{*}=\bar{\varepsilon}_{s}^{*}$. The explicit description of the maps is as follows:

$$
\begin{array}{ll}
\varepsilon_{t}(h)=\left\langle\varepsilon, 1_{(1)} h\right\rangle 1_{(2)} & \varepsilon_{s}(h)=\left\langle\varepsilon, h 1_{(2)}\right\rangle 1_{(1)} \\
\bar{\varepsilon}_{t}(h)=\left\langle\varepsilon, h 1_{(1)}\right\rangle 1_{(2)} & \bar{\varepsilon}_{s}(h)=\left\langle\varepsilon, 1_{(2)} h\right\rangle 1_{(1)}
\end{array}
$$

Lemma 1.2. Let $H$ be a prebialgebra. Then we have the following properties, for all $h, g \in H$ :

$$
\begin{aligned}
(\mathrm{rm}) & \Longleftrightarrow h \varepsilon_{t}(g)=\left\langle\varepsilon, h_{(1)} g\right\rangle h_{(2)} \\
(\mathrm{rm}) & \Longleftrightarrow \varepsilon_{s}(g) h=\left\langle\varepsilon, g h_{(2)}\right\rangle h_{(1)} \\
(\mathrm{lm}) & \Longleftrightarrow \bar{\varepsilon}_{t}(g) h=\left\langle\varepsilon, g h_{(1)}\right\rangle h_{(2)} \\
(\mathrm{lm}) & \Longleftrightarrow h \bar{\varepsilon}_{s}(g)=\left\langle\varepsilon, h_{(2)} g\right\rangle h_{(1)} \\
(\mathrm{rc}) & \Longleftrightarrow h_{(1)} \otimes \varepsilon_{t}\left(h_{(2)}\right)=1_{(1)} h \otimes 1_{(2)} \\
(\mathrm{rc}) & \Longleftrightarrow \varepsilon_{s}\left(h_{(1)}\right) \otimes h_{(2)}=1_{(1)} \otimes h 1_{(2)} \\
(\mathrm{lc}) & \Longleftrightarrow h_{(1)} \otimes \bar{\varepsilon}_{t}\left(h_{(2)}\right)=h 1_{(1)} \otimes 1_{(2)} \\
(\mathrm{lc}) & \Longleftrightarrow \bar{\varepsilon}_{s}\left(h_{(1)}\right) \otimes h_{(2)}=1_{(1)} \otimes 1_{(2)} h
\end{aligned}
$$

We introduce several subspaces of $H$ and $H^{*}$.

$$
\begin{array}{rlll}
\operatorname{Im}(f)=H_{L} & \operatorname{Im}\left(f^{\prime}\right)=H_{R} & \operatorname{Im}(g)=H_{L}^{*} & \operatorname{Im}\left(g^{\prime}\right)=H_{R}^{*} \\
\operatorname{Im}\left(\varepsilon_{t}\right)=H_{t} & \operatorname{Im}\left(\varepsilon_{s}\right)=H_{s} & \operatorname{Im}\left(\varepsilon_{t}^{*}\right)=H_{t}^{*} & \operatorname{Im}\left(\varepsilon_{s}^{*}\right)=H_{s}^{*} \\
\operatorname{Im}\left(\bar{\varepsilon}_{t}\right)=\bar{H}_{t} & \operatorname{Im}\left(\bar{\varepsilon}_{s}\right)=\bar{H}_{s} & \operatorname{Im}\left(\bar{\varepsilon}_{t}^{*}\right)=\bar{H}_{t}^{*} & \operatorname{Im}\left(\bar{\varepsilon}_{s}^{*}\right)=\bar{H}_{s}^{*} \\
I_{t}=\left\{h \in H \mid \Delta(h)=1_{(1)} h \otimes 1_{(2)}\right\} & I_{s}=\left\{h \in H \mid \Delta(h)=1_{(1)} \otimes h 1_{(2)}\right\} \\
\bar{I}_{t}=\left\{h \in H \mid \Delta(h)=h 1_{(1)} \otimes 1_{(2)}\right\} & \bar{I}_{s}=\left\{h \in H \mid \Delta(h)=1_{(1)} \otimes 1_{(2)} h\right\} \\
I_{t}^{*}=\left\{h^{*} \in H^{*} \mid\left\langle h^{*}, k l\right\rangle=\left\langle\varepsilon, k l_{(2)}\right\rangle\left\langle h^{*}, l_{(1)}\right\rangle\right\} \\
I_{s}^{*}=\left\{h^{*} \in H^{*} \mid\left\langle h^{*}, k l\right\rangle=\left\langle\varepsilon, k_{(1)} l\right\rangle\left\langle h^{*}, k_{(2)}\right\rangle\right\} \\
\bar{I}_{t}^{*}=\left\{h^{*} \in H^{*} \mid\left\langle h^{*}, k l\right\rangle=\left\langle\varepsilon, k l_{(1)}\right\rangle\left\langle h^{*}, l_{(2)}\right\rangle\right\} \\
\bar{I}_{s}^{*}=\left\{h^{*} \in H^{*} \mid\left\langle h^{*}, k l\right\rangle=\left\langle\varepsilon, k_{(2)} l\right\rangle\left\langle h^{*}, k_{(1)}\right\rangle\right\}
\end{array}
$$


Lemma 1.3. Let $H$ be a prebialgebra. Then

$$
\begin{gathered}
I_{t} \subset H_{t} \subset H_{L} \supset \bar{H}_{t} \supset \bar{I}_{t} \quad ; \quad I_{s} \subset H_{s} \subset H_{R} \supset \bar{H}_{s} \supset \bar{I}_{s} \\
I_{t}^{*} \subset H_{t}^{*} \subset H_{L}^{*} \supset \bar{H}_{t}^{*} \supset \bar{I}_{t}^{*} ; I_{s}^{*} \subset H_{s}^{*} \subset H_{R}^{*} \supset \bar{H}_{s}^{*} \supset \bar{I}_{s}^{*} \\
(\mathrm{rc}) \Longrightarrow I_{t}=H_{t}=H_{L} ; I_{s}=H_{s}=H_{R} ; I_{t}^{*}=H_{t}^{*} ; I_{s}^{*}=H_{s}^{*} ; \\
(\mathrm{lc}) \Longrightarrow \bar{I}_{t}=\bar{H}_{t}=H_{L} ; \bar{I}_{s}=\bar{H}_{s}=H_{R} ; \bar{I}_{t}^{*}=\bar{H}_{t}^{*} ; \bar{I}_{s}^{*}=\bar{H}_{s}^{*} ; \\
(\mathrm{rm}) \Longrightarrow I_{t}^{*}=H_{t}^{*}=H_{L}^{*} ; I_{s}^{*}=H_{s}^{*}=H_{R}^{*} ; I_{t}=H_{t} ; I_{s}=H_{s} ; \\
(\mathrm{lm}) \Longrightarrow \bar{I}_{t}^{*}=\bar{H}_{t}^{*}=H_{L}^{*} ; \bar{I}_{s}^{*}=\bar{H}_{s}^{*}=H_{R}^{*} ; \bar{I}_{t}=\bar{H}_{t} ; \bar{I}_{s}=\bar{H}_{s} .
\end{gathered}
$$

Consequently

$$
\begin{aligned}
&(\mathrm{c}) \Longrightarrow H_{t}=\bar{H}_{t} ; H_{s}=\bar{H}_{s} ; \\
&(\mathrm{m}) \Longrightarrow H_{t}^{*}=\bar{H}_{t}^{*} ; H_{s}^{*}=\bar{H}_{s}^{*} .
\end{aligned}
$$

Proof. We first prove the statements relating $I_{t}, H_{t}$ and $H_{L}$. If $h \in I_{t}$, then $\varepsilon_{t}(h)=\varepsilon\left(1_{(1)} h\right) 1_{(2)}=\varepsilon\left(h_{(1)}\right) h_{(2)}=h$, hence $h \in H_{t}$.

Obviously $H_{t}=\operatorname{Im}(f \circ g) \subset \operatorname{Im}(f)=H_{L}$.

Assume that (rc) holds, and take $h=f\left(h^{*}\right)=\left\langle h^{*}, 1_{(1)}\right\rangle 1_{(2)} \in H_{L}=\operatorname{Im}(f)$. Then $\Delta(h)=\left\langle h^{*}, 1_{(1)}\right\rangle 1_{\left(1^{\prime}\right)} 1_{(2)} \otimes 1_{\left(2^{\prime}\right)}=1_{\left(1^{\prime}\right)} h \otimes 1_{\left(2^{\prime}\right)}$, hence $h \in I_{t}$.

Now assume that (rm) holds, and take $h=\varepsilon_{t}(k) \in H_{t}$. Then $\Delta(h)=$ $\varepsilon\left(1_{(1)} k\right) \Delta\left(1_{(2)}\right)=\varepsilon\left(1_{(1)} k\right) 1_{(2)} \otimes 1_{(3)} \stackrel{=1}{=} 1_{(1)} \varepsilon_{t}(k) \otimes 1_{(2)}=1_{(1)} h \otimes 1_{(2)}$, and it follows that $h \in I_{t}$.

If $H$ is finite, then the statements relating $I_{t}^{*}, H_{t}^{*}$ and $H_{L}^{*}$ follow by applying the statements relating $I_{t}, H_{t}$ and $H_{L}$ to the prebialgebra $H^{*}$. They can be proved easily without the finiteness assumption. First assume that $h^{*} \in I_{t}^{*}$. Then for all $h \in H$, we have that $\left\langle\varepsilon_{t}^{*}\left(h^{*}\right), h\right\rangle=\left\langle h^{*}, \varepsilon_{s}(h)\right\rangle=$ $\left\langle\varepsilon, h 1_{(2)}\right\rangle\left\langle h^{*}, 1_{(1)}\right\rangle=\left\langle h^{*}, h 1\right\rangle=\left\langle h^{*}, h\right\rangle$, hence $\varepsilon_{t}^{*}\left(h^{*}\right)=h^{*} \in H_{t}^{*}$.

Obviously $H_{t}^{*}=\operatorname{Im}(g \circ f) \subset \operatorname{Im}(g)=H_{L}^{*}$.

Now assume that $(\mathrm{rm})$ holds, and take $h^{*} \in \operatorname{Im}(g)=H_{L}^{*}$. Then there exists $h \in H$ such that $\left\langle h^{*}, k\right\rangle=\langle\varepsilon, k h\rangle$, for all $k \in H$. Then we have for all $k, l \in H$ that $\left\langle h^{*}, k l\right\rangle=\langle\varepsilon, k l h\rangle \stackrel{(\mathrm{rm})}{=}\left\langle\varepsilon, k l_{(2)}\right\rangle\left\langle\varepsilon, l_{(1)} h\right\rangle=\left\langle\varepsilon, k l_{(2)}\right\rangle\left\langle h^{*}, l_{(1)}\right\rangle$ and this means that $h^{*} \in I_{t}^{*}$.

Now assume that (rc) holds, and take $\varphi \in H_{t}^{*}$. This means that there exists $h^{*} \in H^{*}$ such that $\langle\varphi, h\rangle=\left\langle h^{*}, \varepsilon_{s}(h)\right\rangle=\left\langle\varepsilon, h 1_{(2)}\right\rangle\left\langle h^{*}, 1_{(1)}\right\rangle$, and then we have for all $k, l \in H$ that

$$
\langle\varphi, k l\rangle=\left\langle\varepsilon, k l 1_{(2)}\right\rangle\left\langle h^{*}, 1_{(1)}\right\rangle \stackrel{\underline{6}}{=}\left\langle\varepsilon, k l_{(2)}\right\rangle\left\langle h^{*}, \varepsilon_{s}\left(l_{(1)}\right)\right\rangle=\left\langle\varepsilon, k l_{(2)}\right\rangle\left\langle\varphi, l_{(1)}\right\rangle,
$$

and this implies that $\varphi \in I_{t}^{*}$. The proof of all the other inclusions is similar.

Note that, in fact, in the cases of (11) and (12), also the opposite implications $I_{t}^{*}=H_{L}^{*} \Rightarrow(\mathrm{rm}) \Leftarrow I_{s}^{*}=H_{R}^{*}$ and $\bar{I}_{t}^{*}=H_{L}^{*} \Rightarrow(\mathrm{lm}) \Leftarrow \bar{I}_{s}^{*}=H_{R}^{*}$ hold. Recall that a $k$-module $M$ is called locally projective if for each finite subset $\left\{m_{1}, \cdots, m_{N}\right\} \subset M$, there exist finite subsets $\left\{e_{1}^{*}, \cdots, e_{m}^{*}\right\} \subset M^{*}$ and $\left\{e_{1}, \cdots, e_{m}\right\} \subset M$ such that $m_{i}=\sum_{j=1}^{m}\left\langle e_{j}^{*}, m_{i}\right\rangle e_{j}$, for all $i \in\{1, \cdots, N\}$. 
It is then easy to show that the natural map $\alpha: M \otimes N \rightarrow \operatorname{Hom}\left(M^{*}, N\right)$, $\alpha(m \otimes n)\left(m^{*}\right)=\left\langle m^{*}, m\right\rangle n$ is injective, for every $k$-module $N$, in other words, $M$ satisfies the $\alpha$-condition. Applying this property, it is easy to show that, in the case where $H$ is locally projective, $I_{t}=H_{L} \Rightarrow(\mathrm{rc}) \Leftarrow I_{s}=H_{R}$ and $\bar{I}_{t}=H_{L} \Rightarrow(\mathrm{lc}) \Leftarrow \bar{I}_{s}=H_{R}$.

Lemma 1.4. Let $H$ be a prebialgebra. If ( $r m$ ) or (rc) holds, then the maps $\varepsilon_{t}, \varepsilon_{s}, \varepsilon_{t}^{*}$ and $\varepsilon_{s}^{*}$ are idempotent; if (lm) or (lc) holds, then the maps $\bar{\varepsilon}_{t}, \bar{\varepsilon}_{s}$, $\bar{\varepsilon}_{t}^{*}$ and $\bar{\varepsilon}_{s}^{*}$ are idempotent.

Lemma 1.5. In any prebialgebra $H$, the following identities hold.

$$
\begin{aligned}
& \varepsilon_{s}\left(1_{(1)}\right) \otimes 1_{(2)}=1_{(1)} \otimes \varepsilon_{t}\left(1_{(2)}\right) ; \quad \bar{\varepsilon}_{s}\left(1_{(1)}\right) \otimes 1_{(2)}=1_{(1)} \otimes \bar{\varepsilon}_{t}\left(1_{(2)}\right) ; \\
& \varepsilon_{t}\left(1_{(1)}\right) \otimes 1_{(2)}=1_{(2)} \otimes \bar{\varepsilon}_{t}\left(1_{(1)}\right) ; \quad \bar{\varepsilon}_{s}\left(1_{(2)}\right) \otimes 1_{(1)}=1_{(1)} \otimes \varepsilon_{s}\left(1_{(2)}\right) .
\end{aligned}
$$

Lemma 1.6. Let $H$ be a prebialgebra.

$$
\begin{aligned}
(\mathrm{rm}) & \Longrightarrow \varepsilon_{t}(h) \varepsilon_{t}(g)=\varepsilon_{t}\left(\varepsilon_{t}(h) g\right) \text { and } \varepsilon_{s}(h) \varepsilon_{s}(g)=\varepsilon_{s}\left(h \varepsilon_{s}(g)\right) \\
(\mathrm{lm}) & \Longrightarrow \bar{\varepsilon}_{t}(h) \bar{\varepsilon}_{t}(g)=\bar{\varepsilon}_{t}\left(h \bar{\varepsilon}_{t}(g)\right) \text { and } \bar{\varepsilon}_{s}(h) \bar{\varepsilon}_{s}(g)=\bar{\varepsilon}_{s}\left(\bar{\varepsilon}_{s}(h) g\right) \\
(\mathrm{rc}) & \Longrightarrow \varepsilon_{t}^{*}\left(h^{*}\right) \varepsilon_{t}^{*}\left(g^{*}\right)=\varepsilon_{t}^{*}\left(\varepsilon_{t}^{*}\left(h^{*}\right) g^{*}\right) \text { and } \varepsilon_{s}^{*}\left(h^{*}\right) \varepsilon_{s}^{*}\left(g^{*}\right)=\varepsilon_{s}^{*}\left(h^{*} \varepsilon_{s}^{*}\left(g^{*}\right)\right) \\
(\mathrm{lc}) & \Longrightarrow \bar{\varepsilon}_{t}^{*}\left(h^{*}\right) \bar{\varepsilon}_{t}^{*}\left(g^{*}\right)=\bar{\varepsilon}_{t}^{*}\left(h^{*} \bar{\varepsilon}_{t}^{*}\left(g^{*}\right)\right) \text { and } \bar{\varepsilon}_{s}^{*}\left(h^{*}\right) \bar{\varepsilon}_{s}^{*}\left(g^{*}\right)=\bar{\varepsilon}_{s}^{*}\left(\bar{\varepsilon}_{s}^{*}\left(h^{*}\right) g^{*}\right)
\end{aligned}
$$

Consequently $H_{t}$ and $H_{s}$ are subalgebras of $H$ if $(r m)$ holds; $\bar{H}_{t}$ and $\bar{H}_{s}$ are subalgebras if (lm) holds; $H_{t}^{*}$ and $H_{s}^{*}$ are subalgebras of $H^{*}$ if $(\mathrm{rc})$ holds; $\bar{H}_{t}^{*}$ and $\bar{H}_{s}^{*}$ are subalgebras of $H^{*}$ if (lc) holds.

Proof. Assume that $(\mathrm{rm})$ holds; then $I_{t}=H_{t}$, and we have for all $h, g \in H$ that $\varepsilon_{t}(h) \varepsilon_{t}(g) \stackrel{\equiv}{=}\left\langle\varepsilon, \varepsilon_{t}(h)_{(1)} g\right\rangle \varepsilon_{t}(h)_{(2)}=\left\langle\varepsilon, 1_{(1)} \varepsilon_{t}(h) g\right\rangle 1_{(2)}=\varepsilon_{t}\left(\varepsilon_{t}(h) g\right)$.

Now assume that (rc) holds. Then

$$
\begin{aligned}
\left\langle\varepsilon_{t}^{*}\left(g^{*}\right)\right. & \left.\varepsilon_{t}^{*}\left(h^{*}\right), h\right\rangle=\left\langle\varepsilon_{t}^{*}\left(g^{*}\right), h_{(2)}\right\rangle\left\langle\varepsilon_{t}^{*}\left(h^{*}\right), h_{(1)}\right\rangle \\
= & \left\langle\varepsilon_{t}^{*}\left(g^{*}\right), h_{(2)}\right\rangle\left\langle h^{*}, \varepsilon_{s}\left(h_{(1)}\right)\right\rangle \underline{\underline{6}}=\left\langle\varepsilon_{t}^{*}\left(g^{*}\right), h 1_{(2)}\right\rangle\left\langle h^{*}, 1_{(1)}\right\rangle \\
& \stackrel{(*)}{=}\left\langle\varepsilon, h 1_{(3)}\right\rangle\left\langle\varepsilon_{t}^{*}\left(g^{*}\right), 1_{(2)}\right\rangle\left\langle h^{*}, 1_{(1)}\right\rangle=\left\langle\varepsilon, h 1_{(2)}\right\rangle\left\langle\varepsilon_{t}^{*}\left(g^{*}\right) h^{*}, 1_{(1)}\right\rangle \\
& =\left\langle\varepsilon_{t}^{*}\left(g^{*}\right) h^{*}, \varepsilon_{s}(h)\right\rangle=\left\langle\varepsilon_{t}^{*}\left(\varepsilon_{t}^{*}\left(g^{*}\right) h^{*}\right), h\right\rangle .
\end{aligned}
$$

$(*)$ : we used that $\varepsilon_{t}^{*}\left(g^{*}\right) \in I_{t}^{*}$, see (9). The proof of all the other assertions is similar.

There are more distinguished subalgebras than those in the previous lemma:

Lemma 1.7. Let $H$ be a prebialgebra.

$(\mathrm{rc}) \Longrightarrow H_{s}$ and $H_{t}$ are subalgebras of $H$

(lc) $\Longrightarrow \bar{H}_{s}$ and $\bar{H}_{t}$ are subalgebras of $H$

$(\mathrm{rm}) \Longrightarrow H_{s}^{*}$ and $H_{t}^{*}$ are subalgebras of $H^{*}$

$(\mathrm{lm}) \Longrightarrow \bar{H}_{s}^{*}$ and $\bar{H}_{t}^{*}$ are subalgebras of $H^{*}$ 
Proof. Assume first that (rc) holds. Then for any $h, k \in H$,

$$
\begin{aligned}
& \varepsilon_{t}(h) \underline{\varepsilon_{f}}(k)=\left\langle\varepsilon, 1_{(1)} k\right\rangle \varepsilon_{t}(h) 1_{(2)} \stackrel{\underline{\underline{6}}}{=}\left\langle\varepsilon, \varepsilon_{s}\left(\varepsilon_{t}(h)_{(1)}\right) k\right\rangle \varepsilon_{t}(h)_{(2)} \\
& \stackrel{\underline{=}}{=}\left\langle\varepsilon, \varepsilon_{s}\left(1_{(1)} \varepsilon_{t}(h)\right) k\right\rangle 1_{(2)}=\left\langle\varepsilon, 1_{\left(1^{\prime}\right)} k\right\rangle\left\langle\varepsilon, 1_{\left(1^{\prime \prime}\right)} h\right\rangle\left\langle\varepsilon, 1_{(1)} 1_{\left(2^{\prime \prime}\right)} 1_{\left(2^{\prime}\right)}\right\rangle 1_{(2)} \\
& \stackrel{(\mathrm{rc})}{=}\left\langle\varepsilon, 1_{\left(1^{\prime}\right)} k\right\rangle\left\langle\varepsilon, 1_{(1)} h\right\rangle\left\langle\varepsilon, 1_{(2)} 1_{\left(2^{\prime}\right)}\right\rangle 1_{(3)}=\left\langle\varepsilon, \varepsilon_{s}\left(1_{(2)}\right) k\right\rangle\left\langle\varepsilon, 1_{(1)} h\right\rangle 1_{(3)} \\
&=\left\langle\left(\varepsilon_{t}^{*} \circ g\right)(k), 1_{(2)}\right\rangle\left\langle g(h), 1_{(1)}\right\rangle 1_{(3)}=f\left(\left(\varepsilon_{t}^{*} \circ g\right)(k) g(h)\right) .
\end{aligned}
$$

This is an element of $H_{L}$, hence by (9) an element of $H_{t}$. Assume next that (rm) holds. Then for any $h^{*}, k^{*} \in H^{*}$ and $h \in H$,

$$
\begin{aligned}
& \left\langle\varepsilon_{t}^{*}\left(h^{*}\right) \varepsilon_{t}^{*}\left(k^{*}\right), h\right\rangle=\left\langle h^{*}, \varepsilon_{s}\left(h_{(2)}\right)\right\rangle\left\langle k^{*}, \varepsilon_{s}\left(h_{(1)}\right)\right\rangle \\
& \quad=\left\langle h^{*}, \varepsilon_{s}\left(h_{(2)}\right)\right\rangle\left\langle k^{*}, 1_{(1)}\right\rangle\left\langle\varepsilon, h_{(1)} 1_{(2)}\right\rangle\left\langle h^{*}, \varepsilon_{s}\left(h \varepsilon_{t}\left(1_{(2)}\right)\right\rangle\left\langle k^{*}, 1_{(1)}\right\rangle\right. \\
& =\left\langle h^{*}, 1_{\left(1^{\prime}\right)}\right\rangle\left\langle\varepsilon, h \varepsilon_{t}\left(1_{(2)}\right) 1_{\left(2^{\prime}\right)}\right\rangle\left\langle k^{*}, 1_{(1)}\right\rangle=\left\langle\varepsilon, h\left(\varepsilon_{t} \circ f\right)\left(k^{*}\right) f\left(h^{*}\right)\right\rangle \\
& \quad=\left\langle g\left(\left(\varepsilon_{t} \circ f\right)\left(k^{*}\right) f\left(h^{*}\right)\right), h\right\rangle .
\end{aligned}
$$

This shows that $\varepsilon_{t}^{*}\left(h^{*}\right) \varepsilon_{t}^{*}\left(k^{*}\right)$ is an element of $H_{L}^{*}$, hence by (11) an element of $H_{t}^{*}$. The rest of the proof is analogous.

If (rm) or (rc) holds, then $\varepsilon_{s}$ and $\varepsilon_{t}$ are projections, hence $H_{s}$ and $H_{t}$ are direct summands of $H$, and $H_{s} \otimes H_{t}$ is a direct summand of $H \otimes H$; moreover $H_{s} \otimes H_{t}=\operatorname{Im}\left(\varepsilon_{s} \otimes \varepsilon_{t}\right)$. In a similar way, if (lm) or (lc) holds, then $\bar{H}_{s} \otimes \bar{H}_{t}=$ $\operatorname{Im}\left(\bar{\varepsilon}_{s} \otimes \bar{\varepsilon}_{t}\right)$. If $(\mathrm{rc})$ holds, then we easily show that $\left(\varepsilon_{s} \otimes \varepsilon_{t}\right)(\Delta(1))=\Delta(1)$, so that we have the following result.

Lemma 1.8. Let $H$ be a prebialgebra.

$$
(\mathrm{rc}) \Longrightarrow \Delta(1) \in H_{s} \otimes H_{t} ;(\mathrm{lc}) \Longrightarrow \Delta(1) \in \bar{H}_{s} \otimes \bar{H}_{t} .
$$

The dual version of Lemma 1.8 is slightly more involved. If (rm) or (rc) holds, then $H_{s}^{*}$ and $H_{t}^{*}$ are direct summands of $H^{*}, H_{s}^{*} \otimes H_{t}^{*}$ is a direct summand of $H^{*} \otimes H^{*}$, and $H_{s}^{*} \otimes H_{t}^{*}=\operatorname{Im}\left(\varepsilon_{s}^{*} \otimes \varepsilon_{t}^{*}\right)=\operatorname{Im}\left(g^{\prime} \circ f^{\prime} \otimes g \circ f\right) \subset$ $\operatorname{Im}\left(g^{\prime} \otimes g\right)$. The converse implication also holds if $(\mathrm{rm})$ is satisfied: take $g^{\prime}(h) \otimes g(k) \in \operatorname{Im}\left(g^{\prime} \otimes g\right)$. By (11), there exist $h^{*}, k^{*} \in H^{*}$ such that $g^{\prime}(h)=g^{\prime}\left(f^{\prime}\left(h^{*}\right)\right)$ and $g(k)=g\left(f\left(k^{*}\right)\right)$. Then $g^{\prime}(h) \otimes g(k)=g^{\prime}\left(f^{\prime}\left(h^{*}\right)\right) \otimes$ $g\left(f\left(k^{*}\right)\right) \in \operatorname{Im}\left(g^{\prime} \circ f^{\prime} \otimes g \circ f\right)$.

Lemma 1.9 is probably folklore, we include an elementary proof for the sake of completeness.

Lemma 1.9. If $H^{*}$ is locally projective as a $k$-module (this is automatically satisfied in the cases where $k$ is a field or $H$ is finite), then the map

$$
\iota: H^{*} \otimes H^{*} \rightarrow(H \otimes H)^{*},\left\langle\iota\left(h^{*} \otimes k^{*}\right), k \otimes h\right\rangle=\left\langle h^{*}, h\right\rangle\left\langle k^{*}, k\right\rangle
$$

is injective.

Proof. Assume that $\iota\left(\sum_{\alpha} h_{\alpha}^{*} \otimes k_{\alpha}^{*}\right)=0$. Since $H^{*}$ is locally projective, there exist $f_{i} \in H^{* *}$ and $k_{i} \in H^{*}$ such that $h_{\alpha}^{*}=\sum_{i} f_{i}\left(h_{\alpha}^{*}\right) k_{i}$, for each $\alpha$. For all $h, k \in H$, we have that $\sum_{\alpha}\left\langle k_{\alpha}^{*}, k\right\rangle\left\langle h_{\alpha}^{*}, h\right\rangle=0$, hence we have for all $k \in H$ 
that $\sum_{\alpha}\left\langle k_{\alpha}^{*}, k\right\rangle h_{\alpha}^{*}=0$. Then it follows that we have for every index $i$ and for every $k \in H$ that

$$
\sum_{\alpha} f_{i}\left(h_{\alpha}^{*}\right)\left\langle k_{\alpha}^{*}, k\right\rangle=f_{i}\left(\sum_{\alpha}\left\langle k_{\alpha}^{*}, k\right\rangle h_{\alpha}^{*}\right)=0
$$

Therefore $\sum_{\alpha} f_{i}\left(h_{\alpha}^{*}\right) k_{\alpha}^{*}=0$ and

$$
\sum_{\alpha} h_{\alpha}^{*} \otimes k_{\alpha}^{*}=\sum_{i} k_{i} \otimes\left(\sum_{\alpha} f_{i}\left(h_{\alpha}^{*}\right) k_{\alpha}^{*}\right)=0 .
$$

Assume that $H^{*}$ is locally projective, and let

$$
H^{o}=\left\{h^{*} \in H^{*} \mid m^{*}\left(h^{*}\right)=h^{*} \circ m \in \operatorname{Im}(\iota)\right\},
$$

where $m$ is the multiplication in $H$. For $h^{*} \in H^{o}$, we write $\iota^{-1}\left(m^{*}\left(h^{*}\right)\right)=$ $\Delta\left(h^{*}\right)=h_{(1)}^{*} \otimes h_{(2)}^{*}$. Then $\Delta$ is coassociative, see for example [8, Prop. 1.5.3] (at least in the case where $k$ is a field). It is easy to show that $H^{o}$ is closed under opposite convolution multiplication.

Proposition 1.10. Let $H$ be a prebialgebra, and assume that $H^{*}$ is locally projective as a $k$-module. If $(\mathrm{rm})$ holds, then $\varepsilon \in H^{o}, \Delta(\varepsilon) \in H_{s}^{*} \otimes H_{t}^{*}$ and $H^{o}$ is a prebialgebra satisfying $(r c) . H_{s}^{*}$ and $H_{t}^{*}$ are subspaces of $H^{o}$, and $\varepsilon_{s}^{*}$ and $\varepsilon_{t}^{*}$ restrict to projections from $H^{o}$ onto $H_{s}^{*}$ and $H_{t}^{*}$.

If (rc) holds, then $\varepsilon \in H^{o}, \Delta(\varepsilon) \in \bar{H}_{s}^{*} \otimes \bar{H}_{t}^{*}$ and $H^{o}$ is a weak prebialgebra satisfying (lc). $\bar{H}_{s}^{*}$ and $\bar{H}_{t}^{*}$ are subspaces of $H^{o}$, and $\bar{\varepsilon}_{s}^{*}$ and $\bar{\varepsilon}_{t}^{*}$ restrict to projections from $H^{o}$ onto $\bar{H}_{s}^{*}$ and $\bar{H}_{t}^{*}$.

If $H$ is a weak bialgebra, then $H^{o}$ is also a weak bialgebra.

Proof. If (rm) holds, then $\left.\langle\varepsilon, h k\rangle=\left\langle\varepsilon, h 1_{(2)}\right)\right\rangle\left\langle\varepsilon, 1_{(1)} 1_{\left(2^{\prime}\right)}\right\rangle\left\langle\varepsilon, 1_{\left(1^{\prime}\right)} k\right\rangle$, hence

$$
\Delta(\varepsilon)=\left\langle\varepsilon, 1_{(1)} 1_{\left(2^{\prime}\right)}\right\rangle g^{\prime}\left(1_{\left(1^{\prime}\right)}\right) \otimes g\left(1_{(2)}\right) \in \operatorname{Im}\left(g^{\prime} \otimes g\right)=H_{s}^{*} \otimes H_{t}^{*} .
$$

Take $\psi \in H_{s}^{*}=I_{s}^{*}($ see (11) $)$. Then

$$
\langle\psi, k l\rangle=\left\langle\varepsilon, k_{(1)} l\right\rangle\left\langle\psi, k_{(2)}\right\rangle=\left\langle\varepsilon_{(1)}, l\right\rangle\left\langle\psi \varepsilon_{(2)}, k\right\rangle,
$$

hence $\Delta(\psi)=\varepsilon_{(1)} \otimes \psi \varepsilon_{(2)}$ and $\psi \in H^{o}$. Then we compute that $(\Delta \otimes$ $\left.H^{o}\right)\left(\varepsilon_{(1)} \otimes \varepsilon_{(2)}\right)=\varepsilon_{\left(1^{\prime}\right)} \otimes \varepsilon_{(1)} \varepsilon_{\left(2^{\prime}\right)} \otimes \varepsilon_{(2)}$, which proves that $H^{o}$ satisfies (rc).

Lemma 1.11. If ( $\mathrm{rm}$ ) or (rc) holds, then we have isomorphisms

$$
H_{t} \underset{f}{\stackrel{g}{\rightleftarrows}} H_{t}^{*} \text { and } H_{s} \underset{f^{\prime}}{\stackrel{g^{\prime}}{\rightleftarrows}} H_{s}^{*} \text {. }
$$

If $(\mathrm{lm})$ or (lc) holds, then we have isomorphisms

$$
\bar{H}_{t} \underset{f}{\stackrel{g^{\prime}}{\rightleftarrows}} \bar{H}_{s}^{*} \text { and } \bar{H}_{s} \underset{f^{\prime}}{\stackrel{g}{\rightleftarrows}} \bar{H}_{t}^{*} .
$$


Proof. Assume that (rm) or (rc) holds. We first show that $g$ restricts and corestricts to $g: H_{t} \rightarrow H_{t}^{*}$. Take $z \in H_{t}$. Since $f \circ g=\varepsilon_{t}$ is a projection, we have that $g(z)=(g \circ f \circ g)(z)=\varepsilon_{t}^{*}(g(z)) \in H_{t}^{*}$.

In a similar way, $f$ restricts and corestricts to $f: H_{t}^{*} \rightarrow H_{t}$ : for $\varphi \in H_{t}^{*}$, we have $f(\varphi)=(f \circ g \circ f)(\varphi)=\varepsilon_{t}(f(\varphi)) \in H_{t}$.

Finally, for $z \in H_{t}$ and $\varphi \in H_{t}^{*}$, we have $(f \circ g)(z)=\varepsilon_{t}(z)=z$ and $(g \circ f)(\varphi)=\varepsilon_{t}^{*}(\varphi)=\varphi$.

Lemma 1.12. If $(\mathrm{rm})$ or $(\mathrm{rc})$ holds, then we have isomorphisms $H_{s}^{*} \cong\left(H_{t}\right)^{*}$ and $H_{t}^{*} \cong\left(H_{s}\right)^{*}$. Moreover $H_{s}$ and $H_{t}$ are finite with finite dual basis $1_{(1)} \otimes g\left(1_{(2)}\right)$ for $H_{s}$ and $1_{(2)} \otimes g^{\prime}\left(1_{(1)}\right)$ for $H_{t}$.

If (lm) or (lc) holds, then $\bar{H}_{t}^{*} \cong\left(\bar{H}_{t}\right)^{*}$ and $\bar{H}_{s}^{*} \cong\left(\bar{H}_{s}\right)^{*} . \bar{H}_{s}$ and $\bar{H}_{t}$ are finite, with finite dual basis $1_{(1)} \otimes g^{\prime}\left(1_{(2)}\right)$ for $\bar{H}_{s}$ and $1_{(2)} \otimes g\left(1_{(1)}\right)$ for $\bar{H}_{t}$.

Proof. Recall that $H_{s}^{*}=\operatorname{Im}\left(\varepsilon_{s}^{*}\right)$ and $\left(H_{t}\right)^{*}$ is the dual of $H_{t}$. If $(\mathrm{rm})$ or (rc) holds, then $\varepsilon_{t}$ is a projection, and $H=H_{t} \oplus K_{t}$, with $K_{t}=\operatorname{Ker}\left(\varepsilon_{t}\right)$. Take $h^{*} \in H_{s}^{*}$. Then $h^{*}=\varepsilon_{s}^{*}\left(h^{*}\right)=h^{*} \circ \varepsilon_{t}$, hence $h^{*}\left(K_{t}\right)=0$. Conversely, if $h^{*}\left(K_{t}\right)=0$, then for all $h \in H$, we have $\left\langle h^{*}, h\right\rangle=\left\langle h^{*}, \varepsilon_{t}(h)\right\rangle+\left\langle h^{*}, h-\right.$ $\left.\varepsilon_{t}(h)\right\rangle=\left\langle h^{*}, \varepsilon_{t}(h)\right\rangle=\left\langle\varepsilon_{s}^{*}\left(h^{*}\right), h\right\rangle$, hence $h^{*}=\varepsilon_{s}^{*}\left(h^{*}\right) \in H_{s}^{*}$. We conclude that $h^{*} \in H_{s}^{*}$ if and only if $h^{*}\left(K_{t}\right)=0$. Now define $\alpha:\left(H_{t}\right)^{*} \rightarrow H_{s}^{*}$, $\alpha\left(z^{*}\right)=z^{*} \circ \varepsilon_{t}$, and $\beta: H_{s}^{*} \rightarrow\left(H_{t}\right)^{*}, \beta(\varphi)=\varphi_{\mid H_{t}} . \alpha$ and $\beta$ are well-defined and inverse to each other.

If (rc) holds, then $1_{(1)} \otimes 1_{(2)} \in H_{s} \otimes H_{t}$, by Lemma 1.8, and then $1_{(2)} \otimes$ $g^{\prime}\left(1_{(1)}\right) \in H_{t} \otimes H^{*} \cong H_{t} \otimes\left(H_{t}\right)^{*}$, by femma 1.11. If (rm) holds, then $\varepsilon_{t}\left(1_{(2)}\right) \otimes g^{\prime}\left(1_{(1)} \stackrel{1=}{=} 1_{(2)} \otimes\left\langle\varepsilon, \varepsilon_{s}\left(1_{(1)}\right)-\right\rangle \stackrel{=}{=} 1_{(2)} \otimes g^{\prime}\left(1_{(1)}\right)\right.$. Using the fact that $H_{R}^{*}=H_{s}^{*}$, see (11), and that $\varepsilon_{t}$ and $\varepsilon_{s}^{*}$ are projections onto $H_{t}$ and $H_{s}^{*}$, by Lemma 1.4, we now easily obtain $\left(\varepsilon_{t} \otimes \varepsilon_{s}^{*}\right)\left(1_{(2)} \otimes g^{\prime}\left(1_{(1)}\right)\right)=1_{(2)} \otimes g^{\prime}\left(1_{(1)}\right)$, hence $1_{(2)} \otimes g^{\prime}\left(1_{(1)}\right) \in \operatorname{Ker}\left(\right.$ id $\left.-\varepsilon_{t} \otimes \varepsilon_{s}^{*}\right)=H_{t} \otimes H_{s}^{*}$. Finally, we have for all $z \in H_{t}$ that $1_{(2)}\left\langle g^{\prime}\left(1_{(1)}\right), z\right\rangle=1_{(2)}\left\langle\varepsilon, 1_{(1)} z\right\rangle=\varepsilon_{t}(z)=z$.

Lemma 1.13. Let $H$ be a prebialgebra.

$(18)(\operatorname{lm}) \Longrightarrow g \circ \bar{\varepsilon}_{s}=g, g^{\prime} \circ \bar{\varepsilon}_{t}=g^{\prime}, \varepsilon_{t} \circ \bar{\varepsilon}_{s}=\varepsilon_{t}$ and $\varepsilon_{s} \circ \bar{\varepsilon}_{t}=\varepsilon_{s}$;

$(19)(\mathrm{rm}) \Longrightarrow g^{\prime} \circ \varepsilon_{s}=g^{\prime}, g \circ \varepsilon_{t}=g, \bar{\varepsilon}_{t} \circ \varepsilon_{s}=\bar{\varepsilon}_{t}$ and $\bar{\varepsilon}_{s} \circ \varepsilon_{t}=\bar{\varepsilon}_{s}$;

(20) (lc) $\Longrightarrow f \circ \bar{\varepsilon}_{s}^{*}=f, f^{\prime} \circ \bar{\varepsilon}_{t}^{*}=f^{\prime}, \varepsilon_{t}^{*} \circ \bar{\varepsilon}_{s}^{*}=\varepsilon_{t}^{*}$ and $\varepsilon_{s}^{*} \circ \bar{\varepsilon}_{t}^{*}=\varepsilon_{s}^{*}$;

$(21)(\mathrm{rc}) \Longrightarrow f^{\prime} \circ \varepsilon_{s}^{*}=f^{\prime}, f \circ \varepsilon_{t}^{*}=f, \bar{\varepsilon}_{t}^{*} \circ \varepsilon_{s}^{*}=\bar{\varepsilon}_{t}^{*}$ and $\bar{\varepsilon}_{s}^{*} \circ \varepsilon_{t}^{*}=\bar{\varepsilon}_{s}^{*}$.

Proof. If ( $\mathrm{lm})$ holds, then applying $\varepsilon$ to both sides of (44) we obtain $g=g \circ \bar{\varepsilon}_{s}$. Then it follows that $\varepsilon_{t} \circ \bar{\varepsilon}_{s}=f \circ g \circ \bar{\varepsilon}_{s}=f \circ g=\varepsilon_{t}$. The proof of all the other assertions is similar.

The proof of our next lemma is straightforward.

Lemma 1.14. Let $H$ be a prebialgebra.

$$
\begin{aligned}
(\mathrm{c}) & \Longrightarrow \varepsilon_{s}(h) \varepsilon_{t}(k)=\varepsilon_{t}(k) \varepsilon_{s}(h) ; \\
(\mathrm{m}) & \Longrightarrow \varepsilon_{s}\left(h_{(1)}\right) \otimes \varepsilon_{t}\left(h_{(2)}\right)=\varepsilon_{s}\left(h_{(2)}\right) \otimes \varepsilon_{t}\left(h_{(1)}\right) .
\end{aligned}
$$


Proposition 1.15. If $(m)$ holds, then we have anti-algebra isomorphisms

$$
H_{t} \stackrel{\bar{\varepsilon}_{s}}{\underset{\varepsilon_{t}}{\rightleftarrows}} \bar{H}_{s} \text { and } H_{s} \underset{\varepsilon_{s}}{\stackrel{\bar{\varepsilon}_{t}}{\rightleftarrows}} \bar{H}_{t} .
$$

If (c) holds, then we have anti-algebra isomorphisms

$$
H_{s}^{*} \underset{\varepsilon_{s}^{*}}{\stackrel{\bar{\varepsilon}_{t}^{*}}{\rightleftarrows}} \bar{H}_{t}^{*} \text { and } H_{t}^{*} \underset{\varepsilon_{t}^{*}}{\stackrel{\bar{\varepsilon}_{s}^{*}}{\rightleftarrows}} \bar{H}_{s}^{*} .
$$

Proof. Assume that (m) holds. Then $H_{t}^{*}=\bar{H}_{t}^{*}$, see (14). According to Lemma 1.11, we have isomorphisms

$$
H_{t} \underset{f}{\stackrel{g}{\rightleftarrows}} H_{t}^{*}=\bar{H}_{t}^{*} \stackrel{f^{\prime}}{\rightleftarrows} \bar{H}_{s}
$$

Then it suffices to observe that $f^{\prime} \circ g=\bar{\varepsilon}_{s}$ and $f \circ g=\varepsilon_{t}$. Moreover, for any $y, y^{\prime} \in \bar{H}_{s}$,

$$
\begin{aligned}
\varepsilon_{t}(y) \varepsilon_{t}\left(y^{\prime}\right) \stackrel{(*)}{=} & \varepsilon_{t}\left(\varepsilon_{t}(y) y^{\prime}\right)=\left\langle\varepsilon, 1_{(1)} 1_{\left(2^{\prime}\right)} y^{\prime}\right\rangle\left\langle\varepsilon, 1_{\left(1^{\prime}\right)} y\right\rangle 1_{(2)} \\
\stackrel{(12)}{=} & \left\langle\varepsilon, 1_{(1)} y_{(2)}^{\prime}\right\rangle\left\langle\varepsilon, y_{(1)}^{\prime} y\right\rangle 1_{(2)} \stackrel{(r m)}{=}\left\langle\varepsilon, 1_{(1)} y^{\prime} y\right\rangle 1_{(2)}=\varepsilon_{t}\left(y^{\prime} y\right),
\end{aligned}
$$

where we applied Lemma 1.6 at $(*)$.

If (c) holds then for any $\varphi, \varphi^{\prime} \in \bar{H}_{t}^{*}$ and $z \in H_{t}$,

$$
\begin{aligned}
\left\langle\varepsilon_{s}^{*}(\varphi)\right. & \left.\varepsilon_{s}^{*}\left(\varphi^{\prime}\right), z\right\rangle \stackrel{\left(*_{1}\right)}{=}\left\langle\varepsilon_{s}^{*}\left(\varphi \varepsilon_{s}^{*}\left(\varphi^{\prime}\right)\right), z\right\rangle \\
\stackrel{\left(*_{2}\right)}{=} & \left\langle\varphi, z_{(2)}\right\rangle\left\langle\varphi^{\prime}, \varepsilon_{t}\left(z_{(1)}\right)\right\rangle=\left\langle\varphi, z_{(2)}\right\rangle\left\langle\varphi^{\prime}, 1_{(2)}\right\rangle\left\langle\varepsilon, 1_{(1)} z_{(1)}\right\rangle \\
& \stackrel{\left(*_{3}\right)}{=}\left\langle\varphi, 1_{(1)} z\right\rangle\left\langle\varphi^{\prime}, 1_{(2)}\right\rangle \stackrel{(* 4)}{=}\left\langle\varphi, z_{(1)}\right\rangle\left\langle\varphi^{\prime}, z_{(2)}\right\rangle \\
= & \left\langle\varphi^{\prime} \varphi, z\right\rangle \stackrel{\left(*_{2}\right)}{=}\left\langle\varepsilon_{s}^{*}\left(\varphi^{\prime} \varphi\right), z\right\rangle .
\end{aligned}
$$

$\left(*_{1}\right)$ : we applied Lemma [1.6 $\left(*_{2}\right): z \in H_{t} ;\left(*_{3}\right): \varphi \in \bar{H}_{t}^{*}=\bar{I}_{t}^{*} ;\left(*_{4}\right)$ : $z \in H_{t}=I_{t}$. Since by Lemma 1.6 $\varepsilon_{s}^{*}(\varphi) \varepsilon_{s}^{*}\left(\varphi^{\prime}\right)$ is an element of $H_{s}^{*} \cong\left(H_{t}\right)^{*}$, this proves multiplicativity of $\varepsilon_{s}^{*}: \bar{H}_{t}^{*} \rightarrow H_{s}^{*}$. It is an isomorphism by similar considerations as in the first part of the proof.

Lemma 1.16. For a monoidal prebialgebra $H$ and any elements $h, k \in H$, the following identities hold.

$$
\begin{array}{ll}
\bar{\varepsilon}_{s}(h) \varepsilon_{t}(k)=\varepsilon_{t}(k) \bar{\varepsilon}_{s}(h) & 1_{(1)} \bar{\varepsilon}_{s}(h) \otimes 1_{(2)}=1_{(1)} \otimes 1_{(2)} \varepsilon_{t}(h) \\
\varepsilon_{s}(h) \bar{\varepsilon}_{t}(k)=\bar{\varepsilon}_{t}(k) \varepsilon_{s}(h) & \varepsilon_{s}(h) 1_{(1)} \otimes 1_{(2)}=1_{(1)} \otimes \bar{\varepsilon}_{t}(h) 1_{(2)} .
\end{array}
$$

Proof. We prove only (24), (25) is checked symmetrically. As for the first equality,

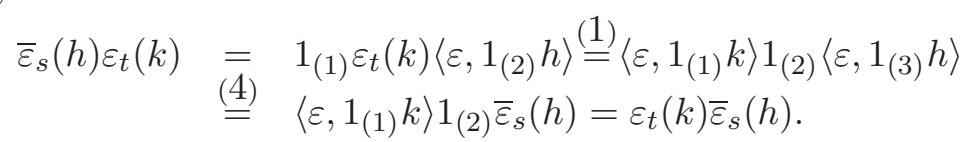


The second equality follows by

$$
1_{(1)} \bar{\varepsilon}_{s}(h) \otimes 1_{(2)} \stackrel{4}{=}\left\langle\varepsilon, 1_{(2)} h\right\rangle 1_{(1)} \otimes 1_{(3)} \stackrel{\underline{1}}{=} 1_{(1)} \otimes 1_{(2)} \varepsilon_{t}(h) .
$$

Let $A$ be a finite algebra, with finite dual basis $\sum_{i} a_{i} \otimes a_{i}^{*} \in A \otimes A^{*}$. It is well-known that $A^{*}$ carries a coalgebra structure, given by the formula

$$
\Delta\left(a^{*}\right)=\sum_{i, j}\left\langle a^{*}, a_{i} a_{j}\right\rangle a_{j}^{*} \otimes a_{i}^{*} .
$$

Proposition 1.17. If any of (rm) and (rc) holds, then $H_{s}$ and $H_{t}$, and also $H_{s}^{*}$ and $H_{t}^{*}$ carry coalgebra structures. If any of $(\mathrm{lm})$ and $(\mathrm{lc})$ holds, then $\bar{H}_{s}$ and $\bar{H}_{t}$, and also $\bar{H}_{s}^{*}$ and $\bar{H}_{t}^{*}$ carry coalgebra structures. The comultiplication maps are given by the formulas

$$
\begin{array}{cl}
\Delta_{s}(y)=\varepsilon_{s}\left(1_{(1)}\right) \otimes \varepsilon_{s}\left(y 1_{(2)}\right) & \Delta_{s}^{*}(\psi)=\varepsilon_{s}^{*}\left(g^{\prime}\left(1_{(1)}\right)\right) \otimes \varepsilon_{s}^{*}\left(\psi g\left(1_{(2)}\right)\right) \\
\Delta_{t}(z)=\varepsilon_{t}\left(1_{(1)} z\right) \otimes \varepsilon_{t}\left(1_{(2)}\right) & \Delta_{t}^{*}(\varphi)=\varepsilon_{t}^{*}\left(g^{\prime}\left(1_{(1)}\right) \varphi\right) \otimes \varepsilon_{t}^{*}\left(g\left(1_{(2)}\right)\right) \\
\bar{\Delta}_{s}(y)=\bar{\varepsilon}_{s}\left(1_{(1)}\right) \otimes \bar{\varepsilon}_{s}\left(1_{(2)} y\right) & \bar{\Delta}_{s}^{*}(\psi)=\bar{\varepsilon}_{s}^{*}\left(\psi g^{\prime}\left(1_{(2)}\right)\right) \otimes \bar{\varepsilon}_{s}^{*}\left(g\left(1_{(1)}\right)\right) \\
\bar{\Delta}_{t}(z)=\bar{\varepsilon}_{t}\left(z 1_{(1)}\right) \otimes \bar{\varepsilon}_{t}\left(1_{(2)}\right) & \bar{\Delta}_{t}^{*}(\varphi)=\bar{\varepsilon}_{t}^{*}\left(g^{\prime}\left(1_{(2)}\right)\right) \otimes \bar{\varepsilon}_{t}^{*}\left(g\left(1_{(1)}\right) \varphi\right)
\end{array}
$$

Proof. Let $H$ be a prebialgebra satisfying (rm) or (rc). From Lemmas 1.6. 1.7 and 1.12, we know that $H_{s}$ and $H_{t}$ are finitely generated projective $k$ algebras, hence $\left(H_{s}\right)^{*} \cong H_{t}^{*}$ and $\left(H_{t}\right)^{*} \cong H_{s}^{*}$ are $k$-coalgebras. The other statements are obtained in a similar way.

The comultiplication maps can be computed easily using the finite dual bases given Lemma 1.12. For example, if ( $\mathrm{rm}$ ) or ( rc) holds, then $1_{(2)} \otimes g^{\prime}\left(1_{(1)}\right) \in$ $H_{t} \otimes H_{s}^{*} \cong H_{t} \otimes\left(H_{t}\right)^{*}$ is a finite dual basis for $H_{t}$, hence the comultiplication on $H_{t}$ is given by

$$
\begin{aligned}
\Delta_{t}(z) & =\left\langle g^{\prime}\left(1_{(1)}\right) g^{\prime}\left(1_{\left(1^{\prime}\right)}\right), z\right\rangle 1_{\left(2^{\prime}\right)} \otimes 1_{(2)} \\
& =\varepsilon_{t}\left(z_{(1)}\right) \otimes \varepsilon_{t}\left(z_{(2)}\right) \stackrel{H_{t}=I_{t}}{=} \varepsilon_{t}\left(1_{(1)} z\right) \otimes \varepsilon_{t}\left(1_{(2)}\right) .
\end{aligned}
$$

The comultiplication on $H_{s}^{*}$ is defined as the transpose of the opposite multiplication in $H_{t}$. That is, for all $z, z^{\prime} \in H_{t}$ and $\psi \in H_{s}^{*} \cong\left(H_{t}\right)^{*}$,

$$
\begin{array}{ll}
\left\langle\Delta_{s}^{*}(\psi), z^{\prime} \otimes z\right\rangle=\left\langle\psi, z z^{\prime}\right\rangle=\left\langle\varepsilon, z_{(1)} z_{(1)}^{\prime}\right\rangle\left\langle\psi, z_{(2)} z_{(2)}^{\prime}\right\rangle \\
\stackrel{H_{t}=I_{t}}{=} & \left\langle\varepsilon, z_{(1)} z^{\prime}\right\rangle\left\langle\psi, z_{(2)}\right\rangle{ }^{z^{\prime} \in H_{t}}\left\langle\varepsilon, 1_{(1)} z^{\prime}\right\rangle\left\langle\varepsilon, z_{(1)} 1_{(2)}\right\rangle\left\langle\psi, z_{(2)}\right\rangle \\
= & \left\langle g^{\prime}\left(1_{(1)}\right) \otimes \psi g\left(1_{(2)}\right), z^{\prime} \otimes z\right\rangle \\
\quad \stackrel{z^{\prime} \in H_{t}}{=} & \left\langle\varepsilon_{s}^{*}\left(g^{\prime}\left(1_{(1)}\right)\right) \otimes \varepsilon_{s}^{*}\left(\psi g\left(1_{(2)}\right)\right), z^{\prime} \otimes z\right\rangle .
\end{array}
$$

Proposition 1.18. Let $H$ be a prebialgebra satisfying $(\mathrm{rm})$ or $(\mathrm{rc})$. Then $\varepsilon_{t}: H \rightarrow H_{t}$ and $\varepsilon_{s}: H \rightarrow H_{s}$ are coalgebra maps. In a similar way, if (lm) or (lc) is satisfied, then $\bar{\varepsilon}_{s}$ and $\bar{\varepsilon}_{t}$ are coalgebra maps.

If $(m)$ holds then $H_{t} \underset{\varepsilon_{t}}{\stackrel{\bar{\varepsilon}_{s}}{\rightleftarrows}} \bar{H}_{s}$ and $H_{s} \underset{\varepsilon_{s}}{\stackrel{\bar{\varepsilon}_{t}}{\rightleftarrows}} \bar{H}_{t}$ are anti-algebra and coalgebra 
isomorphisms.

If (c) holds, then $\bar{H}_{t}=H_{t}, \bar{H}_{s}=H_{s}, \bar{\Delta}_{t}=\Delta_{t}^{\text {cop }}$ and $\bar{\Delta}_{s}=\Delta_{s}^{\text {cop }}$.

Proof. If (rm) holds, then we have for all $h \in H$ that

$$
\begin{aligned}
& \varepsilon_{t}\left(h_{(1)}\right) \otimes \varepsilon_{t}\left(h_{(2)}\right)=\varepsilon_{t}\left(h_{(1)}\right) \otimes\left\langle\varepsilon, 1_{(1)} h_{(2)}\right\rangle 1_{(2)} \stackrel{2}{=} \varepsilon_{t}\left(\varepsilon_{s}\left(1_{(1)}\right) h\right) \otimes 1_{(2)} \\
& \overline{\overline{1}} \varepsilon_{t}\left(1_{(1)} h\right) \otimes \varepsilon_{t}\left(1_{(2)}\right)=\left\langle\varepsilon, 1_{\left(1^{\prime}\right)} 1_{(1)} h\right\rangle 1_{\left(2^{\prime}\right)} \otimes \varepsilon_{t}\left(1_{(2)}\right)
\end{aligned}
$$

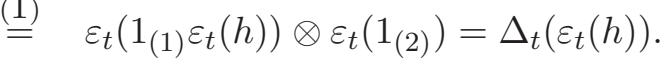

If (rc) holds, then $\varepsilon_{t}$ is idempotent, by Lemma 1.4, and we have for all $h \in H$ that $\varepsilon_{t}\left(h_{(1)}\right) \otimes \varepsilon_{t}\left(h_{(2)}\right) \stackrel{(5)}{=} \varepsilon_{t}\left(1_{(1)} h\right) \otimes \varepsilon_{t}\left(1_{(2)}\right)=\Delta_{t}\left(\varepsilon_{t}(h)\right)$.

Assume that $(\mathrm{m})$ holds. Then the stated maps are anti-algebra isomorphisms by Proposition 1.15. Moreover, the diagram

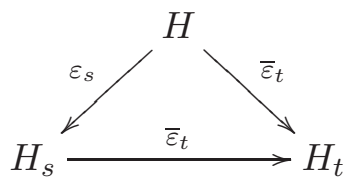

commutes, by (19). Now the two vertical maps in the diagram are coalgebra maps, hence the horizontal map is also a coalgebra map.

Finally, assume that (c) holds. Then

$$
\begin{aligned}
\bar{\Delta}_{t}(z)= & \bar{\varepsilon}_{t}\left(z 1_{(1)}\right) \otimes 1_{(2)}=\left\langle\varepsilon, z 1_{(1)} 1_{\left(1^{\prime}\right)}\right\rangle 1_{\left(2^{\prime}\right)} \otimes 1_{(2)} \\
& =\left\langle\varepsilon, 1_{(1)} 1_{\left(1^{\prime}\right)} z\right\rangle 1_{\left(2^{\prime}\right)} \otimes 1_{(2)}=1_{\left(2^{\prime}\right)} \otimes \varepsilon_{t}\left(1_{\left(1^{\prime}\right)} z\right)=\Delta_{t}^{\mathrm{cop}}(z),
\end{aligned}
$$

where the third equality follows by (17) and (22).

Corollary 1.19. Let $H$ be a prebialgebra satisfying $(\mathrm{rm})$, and assume that $H^{*}$ is locally projective. Then $\varepsilon_{t}^{*}: H^{o} \rightarrow H_{t}^{*}$ and $\varepsilon_{s}^{*}: H^{o} \rightarrow H_{s}^{*}$ are coalgebra maps.

Proof. We have seen in Proposition 1.10 that $H^{o}$ is a prebialgebra satisfying (rc). Then it suffices to apply Proposition 1.18 to $H^{o}$.

\section{The REPRESEntATion CATEGORY}

In this section, we follow the constructions from 13 . Let $H$ be a prebialgebra, and take $M, N \in{ }_{H} \mathcal{M} . M \otimes N$ is a left $H \otimes H$-module, and then an associative but non-unital left $H$-module via restriction of scalars via $\Delta$ :

$$
h \cdot(m \otimes n)=h_{(1)} m \cdot \otimes h_{(2)} \cdot n .
$$

This $H$-action is non-unital in general, since we do not assume the property $\Delta(1)=1 \otimes 1$. However, the $H$-action becomes unital if we restrict $M \otimes N$ to $M \otimes^{l} N=1 \cdot(M \otimes N)$. Then $\otimes^{l}$ is an associative tensor product on ${ }_{H} \mathcal{M}$, the associativity constraint is induced by the natural associativity constraint on the category ${ }_{k} \mathcal{M}$ of $k$-modules. In order to make ${ }_{H} \mathcal{M}$ a monoidal category, 
we need a unit object.

Observe that $H_{L}^{*}=\operatorname{Im}(g)$ is a left $H$-module. Indeed, an element of $\varphi \in H_{L}^{*}$ is of the form $\varphi=h-\varepsilon$, for some $h \in H$, and we define

$$
k \cdot \varphi=(k h) \rightarrow \varepsilon=g(k h) \in H_{L}^{*} .
$$

This $H$-action on $H_{L}^{*}$ is defined in such a way that $g: H \rightarrow H_{L}^{*}$ is left $H$-linear. $H_{L}^{*}$ will be our candidate for unit object. Take $M \in{ }_{H} \mathcal{M}$, and consider the maps

$$
\begin{aligned}
l_{M}: H_{L}^{*} \otimes M \rightarrow M & ; \quad l_{M}(\varphi \otimes m)=f(\varphi) m ; \\
\bar{l}_{M}: M \rightarrow H_{L}^{*} \otimes M & ; \quad \bar{l}_{M}(m)=g\left(1_{(1)}\right) \otimes 1_{(2)} m ; \\
r_{M}: M \otimes H_{L}^{*} \rightarrow M & ; \quad r_{M}(m \otimes \varphi)=f^{\prime}(\varphi) m ; \\
\bar{r}_{M}: M \rightarrow M \otimes^{l} H_{L}^{*} & ; \quad \bar{r}_{M}(m)=1_{(1)} m \otimes g\left(1_{(2)}\right) .
\end{aligned}
$$

The restrictions of $l_{M}$ and $r_{M}$ will be the candidates for the left and right unit constraints, and $\bar{l}_{M}$ and $\bar{r}_{M}$ for their inverses. For all $m \in M$, we have that

$$
\left(l_{M} \circ \bar{l}_{M}\right)(m)=\left(r_{M} \circ \bar{r}_{M}\right)(m)=m .
$$

Proposition 2.1. For a prebialgebra $H$, the following conditions are equivalent:

(1) $\bar{l}_{M}\left(\right.$ resp. $\left.\bar{r}_{M}\right)$ is left $H$-linear, for all $M \in{ }_{H} \mathcal{M}$;

(2) $\bar{l}_{H}\left(\right.$ resp. $\left.\bar{r}_{H}\right)$ is left $H$-linear;

(3) for all $h \in H$, we have that $g\left(1_{(1)}\right) \otimes 1_{(2)} h=g\left(h_{(1)}\right) \otimes h_{(2)}$ (resp. $\left.1_{(1)} h \otimes g\left(1_{(2)}\right)=h_{(1)} \otimes g\left(h_{(2)}\right)\right) ;$

(4) (lm) (resp. (rm)) holds.

Proof. We only prove the "left" part. 1) $\Rightarrow 2$ ) is trivial.

$2) \Rightarrow 3)$. If $\bar{l}_{H}$ is left $H$-linear, then we have for all $h \in H$ that $g\left(1_{(1)}\right) \otimes$ $\overline{1_{(2)} h=} \bar{l}_{H}(h)=h \cdot \bar{l}_{H}(1)=h_{(1)} g\left(1_{(1)}\right) \otimes h_{(2)} 1_{(2)} 1=g\left(h_{(1)}\right) \otimes h_{(2)}$, and condition 3) follows.

$3) \Rightarrow 1) . \bar{l}_{M}(h m)=g\left(1_{(1)}\right) \otimes 1_{(2)} h m=g\left(h_{(1)}\right) \otimes h_{(2)} m=h \bar{l}_{M}(m)$.

$\overline{3) \Rightarrow 4)}$. Evaluating the first tensor factor of $g\left(1_{(1)}\right) \otimes 1_{(2)} h=g\left(h_{(1)}\right) \otimes h_{(2)}$ at $k \in H$, we obtain

$$
\left\langle\varepsilon, k 1_{(1)}\right\rangle 1_{(2)} h=\left\langle\varepsilon, k h_{(1)}\right\rangle h_{(2)} .
$$

Apply $\varepsilon$ to the second tensor factor of (27):

$$
\left\langle\varepsilon, k 1_{(1)}\right\rangle\left\langle\varepsilon, 1_{(2)} h\right\rangle=\langle\varepsilon, k h\rangle .
$$

(lm) follows after we multiply (27) to the right by $l \in H$, and apply $\varepsilon$ to it:

$$
\langle\varepsilon, k h l\rangle \stackrel{\text { 28) }}{=}\left\langle\varepsilon, k 1_{(1)}\right\rangle\left\langle\varepsilon, 1_{(2)} h l\right\rangle=\left\langle\varepsilon, k h_{(1)}\right\rangle\left\langle\varepsilon, h_{(2)} l\right\rangle .
$$

4) $\Rightarrow 3)$. For all $h \in H$, we have that

$$
\begin{aligned}
\overline{g\left(h_{(1)}\right)} & \stackrel{\otimes}{h_{(2)}} \stackrel{\stackrel{18}{=}}{=} g\left(\bar{\varepsilon}_{s}\left(h_{(1)}\right)\right) \otimes h_{(2)}=g\left(1_{(1)}\right)\left\langle\varepsilon, 1_{(2)} h(1)\right\rangle \otimes h_{(2)} \\
& \stackrel{(1)}{=} g\left(1_{(1)}\right) \otimes \bar{\varepsilon}_{t}\left(1_{(2)}\right) h \stackrel{[15)}{=} g\left(\bar{\varepsilon}_{s}\left(1_{(1)}\right)\right) \otimes 1_{(2)} h \stackrel{(18}{=} g\left(1_{(1)}\right) \otimes 1_{(2)} h .
\end{aligned}
$$


Proposition 2.2. Let $H$ be a monoidal prebialgebra. Then $\bar{l}_{M}: M \rightarrow$ $H_{L}^{*} \otimes^{l} M$ and $\bar{r}_{M}: M \rightarrow M \otimes^{l} H_{L}^{*}$ are bijective, and their inverses are respectively the restriction of $l_{M}$ to $H_{L}^{*} \otimes^{l} M$ and the restriction of $r_{M}$ to $M \otimes^{l} H_{L}^{*}$

Proof. $H_{L}^{*} \otimes^{l} M$ is generated by elements of the form $g\left(1_{(1)} h\right) \otimes 1_{(2)} m$. We first compute that

$$
l_{M}\left(g\left(1_{(1)} h\right) \otimes 1_{(2)} m\right)=(f \circ g)\left(1_{(1)} h\right) 1_{(2)} m=\varepsilon_{t}\left(1_{(1)} h\right) 1_{(2)} m=\varepsilon_{t}(h) m .
$$

then

$$
\begin{gathered}
\bar{l}_{M}\left(\varepsilon_{t}(h) m\right)=g\left(1_{(1)}\right) \otimes 1_{(2)} \varepsilon_{t}(h) m=g\left(\varepsilon_{t}(h)_{(1)}\right) \otimes \varepsilon_{t}(h)_{(2)} m \\
=g\left(1_{(1)} \varepsilon_{t}(h)\right) \otimes 1_{(2)} m=g\left(1_{(1)} h\right) \otimes 1_{(2)} m,
\end{gathered}
$$

where the last equality follows by the identity $g(h k)=\varepsilon(-h k) \stackrel{1}{=} \varepsilon\left(-h \varepsilon_{t}(k)\right)=$ $g\left(h \varepsilon_{t}(k)\right)$, for all $h, k \in H$. This proves that $\bar{l}_{M}$ is a left inverse of $l_{M}$ and we already know from (26) that it is a right inverse.

Theorem 2.3. Let $H$ be a monoidal prebialgebra. Then we have a monoidal category $\left(H \mathcal{M}, \otimes^{l}, H_{L}^{*}, a, l, r\right)$.

Proof. It is clear that $\bar{l}$ and $\bar{r}$ are natural, and then the restrictions of $l$ and $r$ are also natural, by Proposition 2.2. To finish the proof, it suffices to show that the triangle axiom [9, XI.(2.9)] holds. This can be seen as follows: for $M, N \in H \mathcal{M}$ and $1_{(1)} m \otimes 1_{(2)} n \in M \otimes^{l} N$, we have that $\bar{r}_{M}\left(1_{(1)} m\right) \otimes 1_{(2)} n=$ $1_{\left(1^{\prime}\right)} 1_{(1)} m \otimes g\left(1_{\left(2^{\prime}\right)}\right) \otimes 1_{(2)} n=1_{(1)} m \otimes g\left(1_{(2)}\right) \otimes 1_{(3)} n=1_{(1)} m \otimes g\left(1_{\left(1^{\prime}\right)}\right) \otimes$ $1_{\left(2^{\prime}\right)} 1_{(2)} n 1_{(1)} m \otimes \bar{l}_{N}\left(1_{(2)} n\right)$.

The map $\pi: M \otimes N \rightarrow M \otimes^{l} N, \pi(m \otimes n)=1_{(1)} m \otimes 1_{(2)} n$ is a projection. Define $M \otimes_{l} N=M \otimes N / \operatorname{Ker}($ id $-\pi)$. Then the map

$$
\bar{\pi}: M \otimes_{l} N \rightarrow M \otimes^{l} N, \bar{\pi}([m \otimes n])=\pi(m \otimes n)
$$

is a well-defined isomorphism of $k$-modules. The left $H$-action on $M \otimes^{l} N$ can be transported to a left $H$-action on $M \otimes_{l} N$, which is given by the usual formula $h \cdot[m \otimes n]=\left[h_{(1)} m \otimes h_{(2)} n\right]$. This means that the functors $\otimes^{l}$ and $\otimes_{l}$ are isomorphic, so - in the case where $H$ is monoidal - the monoidal structure on ${ }_{H} \mathcal{M}$ can be described by $\otimes_{l}$.

From Lemmas 1.3 and 1.11, we know that if $H$ is a monoidal prebialgebra then $H_{L}^{*}=H_{t}^{*}$ and $f: H_{t}^{*} \rightarrow H_{t}$ is an isomorphism with inverse $g$. So we can transport the left $H$-action on $H_{L}^{*}$ to $H_{t}$, making $H_{t}$ the unit object of ${ }_{H} \mathcal{M}$. This transported action is easily computed: for $h \in H, z \in H_{t}$, we find $h . z=f(h \cdot g(z))=(f \circ g)(h z)=\varepsilon_{t}(h z)$.

Recall that any monoidal functor $U:(\mathcal{M}, \otimes, I) \rightarrow(\mathcal{N}, \times, J)$ factorizes uniquely through a monoidal functor from $\mathcal{M}$ to the bimodule category of the monoid $U(I)$ in $\mathcal{N}$ via the forgetful functor ${ }_{U(I)} \mathcal{N}_{U(I)} \rightarrow \mathcal{N}$. In [18] 
a monoidal functor $U$ was termed essentially strong monoidal whenever the functor $\mathcal{M} \rightarrow_{U(I)} \mathcal{N}_{U(I)}$ in its factorization is strong monoidal. There is an evident dual notion of essentially strong opmonoidal functor.

Theorem 2.4. Let $H$ be a monoidal prebialgebra. Then the forgetful functor $U:{ }_{H} \mathcal{M} \rightarrow{ }_{k} \mathcal{M}$ is monoidal and opmonoidal. If any of (rc) and (lc) holds, then it is essentially strong monoidal and essentially strong opmonoidal.

Proof. The monoidal structure of $U$ is given by

$$
U^{M, N}: M \otimes^{l} N \longrightarrow M \otimes N ; \quad U^{0}: H_{L}^{*} \stackrel{\langle-, 1\rangle}{\longrightarrow} k
$$

and the opmonoidal structure of $U$ is given by

$$
U_{M, N}: M \otimes N \stackrel{\pi}{\longrightarrow} M \otimes^{l} N ; \quad U_{0}: k \stackrel{(-) \varepsilon}{\longrightarrow} H_{L}^{*}
$$

for any left $H$-modules $M$ and $N$. Note that $U_{M, N} \circ U^{M, N}$ is the identity map. These monoidal and opmonoidal structures render $U$ with the structure of a separable Frobenius monoidal functor if and only if $H$ is a weak bialgebra.

The monoidal structure of $U$ induces $H_{t}$-bimodule actions on any left $H$ module $M$. Their explicit form comes out as

$$
z \triangleright m=z \cdot m \quad \text { and } \quad m \triangleleft z=\bar{\varepsilon}_{s}(z) \cdot m .
$$

(Associativity of the right action follows directly by Proposition [1.15] and both actions commute in light of (24)). The opmonoidal structure of $U$ induces $H_{t}$-bicomodule coactions on any left $H$-module $M$. Explicitly,

$$
m \mapsto 1_{(1)} \cdot m \otimes \varepsilon_{t}\left(1_{(2)}\right) \quad \text { and } \quad m \mapsto \varepsilon_{t}\left(1_{(1)}\right) \otimes 1_{(2)} \cdot m .
$$

(Commutativity of these coactions can be verified also directly by

$$
\begin{aligned}
& \varepsilon_{t}\left(1_{\left(\frac{1)}{25)}\right)} \otimes 1_{(2)} 1_{\left(1^{\prime}\right)} \otimes \varepsilon_{t}\left(1_{\left(2^{\prime}\right)}\right)=1_{(2)} \otimes \bar{\varepsilon}_{t}\left(1_{(1)}\right) \varepsilon_{s}\left(1_{\left(1^{\prime}\right)}\right) \otimes 1_{\left(2^{\prime}\right)}\right. \\
& \stackrel{=}{=} 1_{(2)} \otimes \varepsilon_{s}\left(1_{\left(1^{\prime}\right)}\right) \bar{\varepsilon}_{t}\left(1_{(1)}\right) \otimes 1_{\left(2^{\prime}\right)}=\varepsilon_{t}\left(1_{(1)}\right) \otimes 1_{\left(1^{\prime}\right)} 1_{(2)} \otimes \varepsilon_{t}\left(1_{\left(2^{\prime}\right)}\right),
\end{aligned}
$$

where the first and last equalities follow by Lemma 1.5. By similar steps also coassociativity of the coactions can be checked directly.)

The monoidal functor $U:{ }_{H} \mathcal{M} \rightarrow{ }_{k} \mathcal{M}$ factorizes through a monoidal functor $S:{ }_{H} \mathcal{M} \rightarrow{ }_{H_{t}} \mathcal{M}_{H_{t}}$. The binary part of the monoidal structure of $S$ comes out as

$$
S^{M, N}: M \otimes_{H_{t}} N \rightarrow M \otimes^{l} N, \quad m \otimes_{H_{t}} n \mapsto 1_{(1)} \cdot m \otimes 1_{(2)} \cdot n .
$$

(One can check directly that it is well-defined by (24).) In order to prove essentially strong monoidality of $U$, we need to construct the inverse of $S^{M, N}$. As a candidate, consider the restriction $S_{M, N}: M \otimes^{l} N \rightarrow M \otimes_{H_{t}} N$ of the canonical epimorphism $M \otimes N \rightarrow M \otimes_{H_{t}} N$,

$$
S_{M, N}\left(1_{(1)} \cdot m \otimes 1_{(2)} \cdot n\right)=1_{(1)} \cdot m \otimes_{H_{t}} 1_{(2)} \cdot n .
$$


Clearly, for any monoidal prebialgebra $H, S^{M, N} \circ S_{M, N}$ is the identity map. On the other hand, $\left(S_{M, N} \circ S^{M, N}\right)\left(m \otimes_{H_{t}} n\right)=1_{(1)} \cdot m \otimes_{H_{t}} 1_{(2)} \cdot n$. By (17), (rc) implies $1_{(1)} \otimes 1_{(2)} \in H \otimes H_{t}$ hence

$$
1_{(1)} \cdot m \otimes_{H_{t}} 1_{(2)} \cdot n=\bar{\varepsilon}_{s}\left(1_{(2)}\right) 1_{(1)} \cdot m \otimes_{H_{t}} n=m \otimes_{H_{t}} n,
$$

where the second equality follows by $\bar{\varepsilon}_{s}\left(1_{(2)}\right) 1_{(1)}=\left\langle\varepsilon, 1_{\left(2^{\prime}\right)} 1_{(2)}\right\rangle 1_{\left(1^{\prime}\right)} 1_{(1)}=1$. Similarly, by (17), (lc) implies $1_{(1)} \otimes 1_{(2)} \in \bar{H}_{s} \otimes H$ hence

$$
1_{(1)} \cdot m \otimes_{H_{t}} 1_{(2)} \cdot n=m \otimes_{H_{t}} \varepsilon_{t}\left(1_{(1)}\right) 1_{(2)} \cdot n=m \otimes_{H_{t}} n .
$$

This proves that whenever (rc) or (lc) holds, $S^{M, N}$ and $S_{M, N}$ are mutual inverses.

By Lemma 1.5] and by the second equality in (25),

$$
\begin{aligned}
1_{\left(1^{\prime}\right)} 1_{(1)} & \otimes \varepsilon_{t}\left(1_{\left(2^{\prime}\right)}\right) \otimes 1_{(2)}=\varepsilon_{s}\left(1_{\left(1^{\prime}\right)}\right) 1_{(1)} \otimes 1_{\left(2^{\prime}\right)} \otimes 1_{(2)} \\
& =1_{(1)} \otimes 1_{\left(2^{\prime}\right)} \otimes \varepsilon_{t}\left(1_{\left(1^{\prime}\right)}\right) 1_{(2)}=1_{(1)} \otimes \varepsilon_{t}\left(1_{\left(1^{\prime}\right)}\right) \otimes 1_{\left(2^{\prime}\right)} 1_{(2)} .
\end{aligned}
$$

This means that $1_{\left(1^{\prime}\right)} 1_{(1)} \cdot m \otimes \varepsilon_{t}\left(1_{\left(2^{\prime}\right)}\right) \otimes 1_{(2)} \cdot n=1_{(1)} \cdot m \otimes \varepsilon_{t}\left(1_{\left(1^{\prime}\right)}\right) \otimes 1_{\left(2^{\prime}\right)} 1_{(2)} \cdot n$ for all $m \in M$ and $n \in N$, so that $M \otimes^{l} N$ is a subspace of the $H_{t^{-}}$-comodule tensor product $M \otimes^{H_{t}} N$.

The opmonoidal functor $U:{ }_{H} \mathcal{M} \rightarrow{ }_{k} \mathcal{M}$ factorizes through an opmonoidal functor ${ }_{H} \mathcal{M} \rightarrow{ }^{H_{t}} \mathcal{M}^{H_{t}}$. The binary part of the opmonoidal structure of the latter functor comes out as the evident inclusion map $M \otimes^{l} N \rightarrow M \otimes^{H_{t}} N$. Thus in order to prove essentially strong opmonoidality of $U$, we need to show that $M \otimes^{H_{t}} N$ and $M \otimes^{l} N$ are coinciding subspaces of $M \otimes N$. Since $\sum m^{i} \otimes n^{i} \in M \otimes^{H_{t}} N, \sum 1_{(1)} \cdot m^{i} \otimes \varepsilon_{t}\left(1_{(2)}\right) \cdot n^{i}=\sum m^{i} \otimes \varepsilon_{t}\left(1_{(1)}\right) 1_{2} \cdot n^{i}=$ $\sum m^{i} \otimes n^{i} ;$ and $\sum \bar{\varepsilon}_{s}\left(1_{(1)}\right) \cdot m^{i} \otimes 1_{(2)} \cdot n^{i}=\sum \bar{\varepsilon}_{s}\left(1_{(2)}\right) 1_{1} \cdot m^{i} \otimes n^{i}=\sum m^{i} \otimes n^{i}$. This shows that, in light of (17), any of (rc) and (lc) implies that $M \otimes^{H_{t}} N$ and $M \otimes^{l} N$ coincide, so that $U$ is essentially strong opmonoidal.

A particular consequence of this theorem is that, for a monoidal prebialgebra $H$ for which any of (rc) and (lc) holds, and for any left $H$-modules $M$ and $N$, there are isomorphisms (of $H$-modules) $M \otimes_{l} N \cong M \otimes^{l} N \cong M \otimes_{H_{t}} N \cong$ $M \otimes^{H_{t}} N$.

It also follows by Theorem 2.4 that a monoidal prebialgebra $H$ for which any of (rc) and (lc) holds, is a left bialgebroid over $H_{t}$ (and in fact also a right bialgebroid over $H_{s}$ ) - some of the axioms occurred in (24).

\section{The COREPRESENTATION CATEGORY}

In Section 2, we have seen that the category of modules over a monoidal prebialgebra is monoidal. Throughout this section, we assume that $H$ is a comonoidal prebialgebra, that is, it satisfies condition (c). Then $I_{s}=H_{s}=H_{R}=\bar{I}_{s}=\bar{H}_{s}$, by Lemma 1.3. For $y \in H_{s}$, we have that $\Delta(y)=1_{(1)} \otimes y 1_{(2)}=1_{(1)} \otimes 1_{(2)} y \in H_{s} \otimes H$, by (17), hence $H_{s}$ is a right $H$-comodule. 
Suppose that we have a coassociative $H$-coaction on $M$, which is not necessarily counital; then we also have an associative $H^{*}$-action on $M$, given by $h^{*} \cdot m=\left\langle\varepsilon, m_{[1]}\right\rangle m_{[0]}$. If $M, N \in \mathcal{M}^{H}$, then $M \otimes N$ has a coassociative $H$-coaction given by

$$
\rho(m \otimes n)=m_{[0]} \otimes n_{[0]} \otimes m_{[1]} n_{[1]} .
$$

Now define $M \otimes^{r} N=\varepsilon \cdot(M \otimes N)$, which is the submodule of $M \otimes N$ generated by elements of the form $\left\langle\varepsilon, m_{[1]} n_{[1]}\right\rangle m_{[0]} \otimes n_{[0]}$. It is then easy to show that $M \otimes^{r} N$ is a counital right $H$-subcomodule of $M \otimes N$. Then $\otimes^{r}$ is an associative tensor product on $\mathcal{M}^{H}$, and the associativity constraint is trivially induced by the associativity on ${ }_{k} \mathcal{M}$. Now consider the maps

$$
\begin{aligned}
l_{M}: H_{s} \otimes M \rightarrow M & ; \quad l_{M}(y \otimes m)=g^{\prime}(y) \cdot m=\left\langle\varepsilon, y m_{[1]}\right\rangle m_{[0]} ; \\
\bar{l}_{M}: M \rightarrow H_{s} \otimes & ;
\end{aligned}
$$

The description of $\bar{l}$ and $\bar{r}$ in terms of $\varepsilon_{(1)}$ and $\varepsilon_{(2)}$ only makes sense if $H^{*}$ is locally projective; the other description holds in general.

Theorem 3.1. Let $H$ be a comonoidal prebialgebra. Then we have a monoidal category $\left(\mathcal{M}^{H}, \otimes^{r}, H_{s}, a, l, r\right)$.

Proof. We first show that $\bar{l}_{M}$ is right $H$-colinear. For all $m \in M$, we have

$$
\begin{aligned}
& \rho\left(\bar{l}_{M}(m)\right)=\rho\left(\bar{\varepsilon}_{s}\left(m_{[1]}\right) \otimes m_{[0]}\right)=1_{(1)} \otimes m_{[0]} \otimes 1_{(2)} \bar{\varepsilon}_{s}\left(m_{[2]}\right) m_{[1]} \\
& \quad=1_{(1)} \otimes m_{[0]} \otimes 1_{(2)} 1_{\left(1^{\prime}\right)} m_{[1]}\left\langle\varepsilon, 1_{\left(2^{\prime}\right)} m_{[2]}\right\rangle=1_{(1)} \otimes m_{[0]} \otimes 1_{(2)} m_{[1]} \\
& \quad \stackrel{8}{=} \bar{\varepsilon}_{s}\left(m_{[1]}\right) \otimes m_{[0]} \otimes m_{[2]}=\bar{l}\left(m_{[0]}\right) \otimes m_{[1]} .
\end{aligned}
$$

We now show that the restriction of $l_{M}$ to $H_{s} \otimes^{r} M$ is the inverse of $\bar{l}_{M}$. It is easy to see that $\left(l_{M} \circ \bar{l}_{M}\right)(m)=m$, for all $m \in M$. $H_{s} \otimes^{r} M$ is generated by elements of the form $\left\langle\varepsilon, y_{(2)} m_{[1]}\right\rangle y_{(1)} \otimes m_{[0]}$. Now

$$
\begin{aligned}
& l_{M}\left(\left\langle\varepsilon, y_{(2)} m_{[1]}\right\rangle y_{(1)} \otimes m_{[0]}\right)=\left\langle\varepsilon, y_{(1)} m_{[1]}\right\rangle\left\langle\varepsilon, y_{(2)} m_{[2]}\right\rangle m_{[0]}=\left\langle\varepsilon, y m_{[1]}\right\rangle m_{[0]} \\
& \bar{l}_{M}\left(\left\langle\varepsilon, y m_{[1]}\right\rangle m_{[0]}\right)=\left\langle\varepsilon, y m_{[2]}\right\rangle \bar{\varepsilon}_{s}\left(m_{[1]}\right) \otimes m_{[0]} \\
& \quad=\left\langle\varepsilon, y m_{[2]}\right\rangle\left\langle\varepsilon, 1_{(2)} m_{[1]}\right\rangle 1_{(1)} \otimes m_{[0]} \\
& =\left\langle\varepsilon, y 1_{\left(2^{\prime}\right)} m_{[2]}\right\rangle\left\langle\varepsilon, 1_{(2)} 1_{\left(1^{\prime}\right)} m_{[1]}\right\rangle 1_{(1)} \otimes m_{[0]} \\
& \stackrel{(\mathrm{lc})}{=}\left\langle\varepsilon, y 1_{(3)} m_{[2]}\right\rangle\left\langle\varepsilon, 1_{(2)} m_{[1]}\right\rangle 1_{(1)} \otimes m_{[0]}=\left\langle\varepsilon, y 1_{(2)} m_{[1]}\right\rangle 1_{(1)} \otimes m_{[0]} \\
& =\left\langle\varepsilon, y_{(2)} m_{[1]}\right\rangle y_{(1)} \otimes m_{[0]} .
\end{aligned}
$$

Let us now show that the triangle axiom holds.

$$
\begin{aligned}
& \left(M \otimes \bar{l}_{N}\right)\left(\left\langle\varepsilon, m_{[1]} n_{[1]}\right\rangle m_{[0]} \otimes n_{[0]}\right)=\left\langle\varepsilon, m_{[1]} n_{[2]}\right\rangle m_{[0]} \otimes \bar{\varepsilon}_{s}\left(n_{[1]}\right) \otimes n_{[0]} \\
& \left\langle\varepsilon, m_{[1]} 1_{(2)} n_{[1]}\right\rangle m_{[0]} \otimes 1_{(1)} \otimes n_{[0]} \\
& \stackrel{\underline{\underline{6}}}{=}\left\langle\varepsilon, m_{[2]} n_{[1]}\right\rangle m_{[0]} \otimes \varepsilon_{s}\left(m_{[1]}\right) \otimes n_{[0]} \\
& =\left(\bar{r}_{M} \otimes N\right)\left(\left\langle\varepsilon, m_{[1]} n_{[1]}\right\rangle m_{[0]} \otimes n_{[0]}\right) .
\end{aligned}
$$


For $M, N \in \mathcal{M}^{H}$, let $M \otimes_{r} N=(M \otimes N) / \operatorname{Ker}($ id $-\pi)$, where $\pi(m \otimes n)=$ $\varepsilon \cdot(m \otimes n) . \quad \pi$ induces a natural isomorphism between $\otimes_{r}$ and $\otimes^{r}$, the arguments are similar to the arguments following Theorem 2.3 .

Theorem 3.2. Let $H$ be a comonoidal prebialgebra. Then the forgetful functor $U: \mathcal{M}^{H} \rightarrow{ }_{k} \mathcal{M}$ is monoidal and opmonoidal. If any of (rm) and $(\mathrm{lm})$ holds, then it is essentially strong monoidal and essentially strong opmonoidal.

Proof. The monoidal structure of $U$ is given by

$$
U^{M, N}: M \otimes^{r} N \longrightarrow M \otimes N ; \quad U^{0}: H_{s} \stackrel{\langle\varepsilon,-\rangle}{\longrightarrow} k
$$

and the opmonoidal structure is given by

$$
U_{M, N}: M \otimes N \stackrel{\pi}{\longrightarrow} M \otimes^{r} N ; \quad U_{0}: k \stackrel{(-) 1}{\longrightarrow} H_{s},
$$

for any right $H$-comodules $M$ and $N$. Note that $U_{M, N} \circ U^{M, N}$ is the identity map. These monoidal and opmonoidal structures make $U$ a separable Frobenius monoidal functor if and only if $H$ is a weak bialgebra.

The monoidal structure of $U$ induces $H_{s}$-bimodule actions on any right $H$ comodule $M$

$$
y \triangleright m=\left\langle\varepsilon, y m_{[1]}\right\rangle m_{[0]} \quad \text { and } \quad m \triangleleft y=\left\langle\varepsilon, m_{[1]} y\right\rangle m_{[0]}
$$

and the opmonoidal structure of $U$ induces $H_{s}$-bicomodule coactions

$$
m \mapsto m_{[0]} \otimes \varepsilon_{s}\left(m_{[1]}\right) \quad \text { and } \quad m \mapsto \bar{\varepsilon}_{s}\left(m_{[1]}\right) \otimes m_{[0]} .
$$

The monoidal functor $U: \mathcal{M}^{H} \rightarrow{ }_{k} \mathcal{M}$ factorizes through a monoidal functor $S:{ }_{H} \mathcal{M} \rightarrow{ }_{H_{s}} \mathcal{M}_{H_{s}}$. The binary part of the monoidal structure of $S$ comes out as

$$
S^{M, N}: M \otimes_{H_{s}} N \rightarrow M \otimes^{r} N, \quad m \otimes_{H_{s}} n \mapsto\left\langle\varepsilon, m_{[1]} n_{[1]}\right\rangle m_{[0]} \otimes n_{[0]} .
$$

(In order to check directly that it is well-defined, note that - since $I_{s}=$ $H_{s}=\bar{H}_{s}=\bar{I}_{s}-$ the $H$-coactions are $H_{s}$-linear in the sense that $(m \triangleleft y)_{[0]} \otimes(m \triangleleft y)_{[1]}=m_{[0]} \otimes m_{[1]} y \quad$ and $\quad(y \triangleright m)_{[0]} \otimes(y \triangleright m)_{[1]}=m_{[0]} \otimes y m_{[1]}$. Then the projection $\pi: M \otimes N \rightarrow M \otimes^{r} N$ is $H_{s}$-balanced.) In order to prove essentially strong monoidality of $U$, we need to construct the inverse of $S^{M, N}$. As a candidate, consider the restriction $S_{M, N}: M \otimes^{r} N \rightarrow M \otimes_{H_{s}} N$ of the canonical epimorphism $M \otimes N \rightarrow M \otimes_{H_{s}} N$,

$$
S_{M, N}\left(\left\langle\varepsilon, m_{[1]} n_{[1]}\right\rangle m_{[0]} \otimes n_{[0]} b i g r\right)=\left\langle\varepsilon, m_{[1]} n_{[1]}\right\rangle m_{[0]} \otimes_{H_{s}} n_{[0]} .
$$

The composite $S^{M, N} \circ S_{M, N}$ is the identity map on $M \otimes^{r} N$ and $S_{M, N} \circ S^{M, N}$ is equal to the idempotent endomorphism of $M \otimes_{H_{s}} N$ given by

$$
S_{M, N} \circ S^{M, N}\left(m \otimes_{H_{s}} n\right)=\left\langle\varepsilon, m_{[1]} n_{[1]}\right\rangle m_{[0]} \otimes_{H_{s}} n_{[0]} .
$$


If $(\mathrm{rm})$ holds then

$$
\begin{aligned}
& \left\langle\varepsilon, m_{[1]} n_{[1]}\right\rangle m_{[0]} \otimes_{H_{s}} n_{[0]} \stackrel{2}{=}\left\langle\varepsilon, \varepsilon_{s}\left(m_{[1]}\right) n_{[1]}\right\rangle m_{[0]} \otimes_{H_{s}} n_{[0]} \\
& =m_{[0]} \otimes_{H_{s}} \varepsilon_{s}\left(m_{[1]}\right) \triangleright n=m_{[0]} \triangleleft \varepsilon_{s}\left(m_{[1]}\right) \otimes_{H_{s}} n \\
& =m_{[0]}\left\langle\varepsilon, m_{[1]} \varepsilon_{s}\left(m_{[2]}\right)\right\rangle \otimes_{H_{s}} n=m \otimes_{H_{s}} n .
\end{aligned}
$$

If $(\mathrm{lm})$ holds then the same equality follows by (4), proving that $U$ is essentially strong monoidal whenever any of $(\mathrm{lm})$ and $(\mathrm{rm})$ holds.

For any comonoidal prebialgebra $H$,

$$
\begin{aligned}
& \left\langle\varepsilon, m_{[2]} n_{[8]}^{n_{[1]}}\right\rangle m_{[0]} \otimes \varepsilon_{s}\left(m_{[1]}\right) \otimes n_{[0]} \stackrel{[6]}{=}\left\langle\varepsilon, m_{[1]} 1_{(2)} n_{[1]}\right\rangle m_{[0]} \otimes 1_{(1)} \otimes n_{[0]} \\
& \stackrel{\equiv}{=}\left\langle\varepsilon, m_{[1]} n_{[2]}\right\rangle m_{[0]} \otimes \bar{\varepsilon}_{s}\left(n_{[1]}\right) \otimes n_{[0]} \text {. }
\end{aligned}
$$

Thus it follows that $M \otimes^{r} N$ is a subspace of the comodule tensor product $M \otimes^{H_{s}} N$.

The opmonoidal functor $U: \mathcal{M}^{H} \rightarrow{ }_{k} \mathcal{M}$ factorizes through a unique opmonoidal functor $\mathcal{M}^{H} \rightarrow{ }^{H_{s}} \mathcal{M}^{H_{s}}$. The binary part of the opmonoidal structure of the latter functor comes out as as the evident inclusion map $M \otimes^{r} N \rightarrow M \otimes^{H_{s}} N$. Now if (rm) holds then for any $\sum m^{i} \otimes n^{i} \in M \otimes^{H_{s}} N$,

$$
\begin{gathered}
\sum\left\langle\varepsilon, m_{[1]}^{i} n_{[1]}^{i}\right\rangle m_{[0]}^{i} \otimes n_{[0]}^{i} \stackrel{\sqrt[2]{=}}{=} \sum\left\langle\varepsilon, \varepsilon_{s}\left(m_{[1]}^{i}\right) n_{[1]}^{i}\right\rangle m_{[0]}^{i} \otimes n_{[0]}^{i} \\
=\sum\left\langle\varepsilon, \bar{\varepsilon}_{s}\left(n_{[2]}^{i}\right) n_{[1]}^{i}\right\rangle m^{i} \otimes n_{[0]}^{i}=\sum m^{i} \otimes n^{i},
\end{gathered}
$$

so that $M \otimes^{H_{s}} N$ and $M \otimes^{r} N$ are coinciding subspaces of $M \otimes N$. If (lm) holds then the same equality follows by (4), proving that $U$ is essentially strong opmonoidal whenever any of $(\mathrm{lm})$ and $(\mathrm{rm})$ holds.

As a particular consequence of Theorem 3.2, we have the following result: given two right comodules $M$ and $N$ over a comonoidal bialgebra $H$ satisfying $(\mathrm{rm})$ or $(\mathrm{lm})$, we have $H$-colinear isomorphisms $M \otimes_{r} N \cong M \otimes^{r} N \cong$ $M \otimes_{H_{s}} N \cong M \otimes^{H_{s}} N$.

It also follows by Theorem 3.2 that a comonoidal prebialgebra $H$ satisfying $(\mathrm{rm})$ or $(\mathrm{lm})$ is a right bicoalgebroid over $H_{s}$.

\section{Frobenius SEPARABle Algebras}

In this section, we first collect some properties of Frobenius separable algebras. They are well-known, and can be found in the literature in various levels of generality. We give the results with a sketch of proof. Recall that a $k$-algebra is called Frobenius if there exists a Frobenius system $(e, \varepsilon)$, consisting of $e=e^{<1>} \otimes e^{<2>} \in A \otimes A$ and $\varepsilon \in A^{*}$ such that $a e=e a$ for all $a \in A$, and $\varepsilon\left(e^{<1>}\right) e^{<2>}=e^{<1>} \varepsilon\left(e^{<2>}\right)=1$. $A$ is called Frobenius separable if, moreover, $e^{<1>} e^{<2>}=1$.

We have a dual notion for coalgebras. A Frobenius system for a coalgebra $C$ consists of a couple $(\theta, 1)$, with $\theta \in(C \otimes C)^{*}$ and $1 \in C$ satisfying

$$
\theta\left(c \otimes d_{(1)}\right) d_{(2)}=c_{(1)} \theta\left(c_{(2)} \otimes d\right),
$$


and $\theta(1 \otimes c)=\theta(c \otimes 1)=\varepsilon(c)$, for all $c \in C$. C is termed coseparable Frobenius if, moreover, $\theta \circ \Delta=\varepsilon$.

Proposition 4.1. [5, 6, Sec. 5.12], [7, Cor. 2.6], [16, Theorem 1.6] Let $A$ be a $k$-module. There is a one-to-one correspondence between (separable) Frobenius algebra structures on $A$ and (coseparable) Frobenius coalgebra structures on $A$.

Proof. Let $A$ be an algebra with Frobenius system $(e, \varepsilon)$. Then we define a comultiplication $\Delta$ on $A$ by $\Delta(a)=a e=e a$. The counit on $A$ will be $\varepsilon$. $\theta \in(A \otimes A)^{*}$ is defined by $\theta(a \otimes b)=\varepsilon(a b)$. Straightforward computations show that $A$ is then a Frobenius coalgebra with Frobenius system $(\theta, 1)$. Conversely, if $A$ is a Frobenius coalgebra with Frobenius system $(\theta, 1)$, then we define a multiplication on $A$ by the formula

$$
a b=\theta\left(a \otimes b_{(1)}\right) b_{(2)}=a_{(1)} \theta\left(a_{(2)} \otimes b\right) .
$$

1 is a unit for this multiplication, and this makes $A$ into an associative algebra with Frobenius system $(\Delta(1), \varepsilon)$.

It is clear that these two constructions are inverse to each other. Finally observe that $e^{<1>} e^{<2>}=1$ is equivalent to $\theta \circ \Delta=\varepsilon$.

Let $A$ be a coalgebra; for $M \in \mathcal{M}^{A}$ and $N \in{ }^{A} \mathcal{M}$, we can consider the cotensor product

$$
M \otimes{ }^{A} N=\left\{\sum_{i} m_{i} \otimes n_{i} \in M \otimes N \mid \sum_{i} m_{i[0]} \otimes m_{i[1]} \otimes n_{i}=\sum_{i} m_{i} \otimes n_{i[-1]} \otimes n_{i[0]} .\right.
$$

Proposition 4.2. Let $A$ be a Frobenius algebra, and consider the corresponding Frobenius coalgebra structure on A, see Proposition 4.1. Then we have isomorphisms of categories $\mathcal{M}_{A} \cong \mathcal{M}^{A}$ and ${ }_{A} \mathcal{M} \cong{ }^{A} \mathcal{M}$.

Proof. On $M \in \mathcal{M}^{A}$, we define an $A$-action by $m a=m_{[0]} \varepsilon\left(m_{[1]} a\right)$. On $M \in \mathcal{M}_{A}$, we define an $A$-coaction by $\rho(m)=m e^{<1>} \otimes e^{<2>}$.

Let $A$ be a separable Frobenius algebra, and consider $M \in \mathcal{M}_{A} \cong \mathcal{M}^{A}$ and $N \in{ }_{A} \mathcal{M} \cong{ }^{A} \mathcal{M}$. We have a projection $\pi: M \otimes N \rightarrow M \otimes N$, $\pi(m \otimes n)=m e^{<1>} \otimes e^{<2>} n$. It is clear that $\operatorname{Im}(\pi)=\operatorname{Ker}(\mathrm{id}-\pi)$ and $\operatorname{Ker}(\pi)=\operatorname{Im}(\mathrm{id}-\pi)$

Proposition 4.3. [15, Remarks 5.2 and 7.5, Prop. 5.3], [14, p. 164-165] With notation as above, we have

$$
\operatorname{Im}(\pi)=M \otimes^{A} N \cong M \otimes_{A} N=(M \otimes N) / \operatorname{Ker}(\pi) .
$$

Proof. Let $x=\sum_{i} m_{i} \otimes n_{i} \in M \otimes N$. If $x \in M \otimes^{A} N$, then

$$
\sum_{i} m_{i} e^{<1>} \otimes e^{<2>} \otimes n_{i}=\sum_{i} m_{i} \otimes e^{<1>} \otimes e^{<2>} n_{i} .
$$


Multiplying the second and third tensor factor, and using the separability, we find that $x=\pi(x)$, so $x \in \operatorname{Im}(\pi)$. Conversely, if $x \in \operatorname{Im}(\pi)$, then

$$
\begin{aligned}
& \sum_{i} m_{i} \otimes e^{<1>} \otimes e^{<2>} n_{i}=\sum_{i} m_{i} e^{<1^{\prime}>} \otimes e^{<1>} \otimes e^{<2>} e^{<2^{\prime}>} n_{i} \\
& =\sum_{i} m_{i} e^{<1^{\prime}>} \otimes e^{<2^{\prime}>} e^{<1>} \otimes e^{<2>} n_{i} \\
& =\sum_{i} m_{i} e^{<1>} e^{<1^{\prime}>} \otimes e^{<2^{\prime}>} \otimes e^{<2>} n_{i}=\sum_{i} m_{i} e^{<1^{\prime}>} \otimes e^{<2^{\prime}>} \otimes n_{i},
\end{aligned}
$$

and it follows that $x \in M \otimes^{A} N$.

Now let $I$ be the submodule of $M \otimes N$ generated by elements of the form $m a \otimes n-m \otimes a n$. Then $M \otimes_{A} N=(M \otimes N) / I$, so it suffices to show that $I=\operatorname{Ker}(\pi)$. If $x=m a \otimes n-m \otimes a n$, then $\pi(x)=m a e^{<1>} \otimes e^{<2>} n-$ $m e^{<1>} \otimes e^{<2>}$ an $=0$, so $I \subset \operatorname{Ker}(\pi)$. Take $y=m \otimes n-m e^{<1>} \otimes e^{<2>} n \in$ $\operatorname{Ker}(\pi)=\operatorname{Im}(\mathrm{id}-\pi)$. Then $y=m \otimes e^{<1>} e^{<2>} n-m e^{<1>} \otimes e^{<2>} n \in I$. The canonical surjection $M \otimes N \rightarrow(M \otimes N) / \operatorname{Ker}(\pi)$ restricts to a map

$$
p: \operatorname{Im}(\pi) \rightarrow(M \otimes N) / \operatorname{Ker}(\pi) .
$$

$\pi: M \otimes N \rightarrow M \otimes N$ induces a well-defined map

$$
q:(M \otimes N) / \operatorname{Ker}(\pi) \rightarrow \operatorname{Im}(\pi) .
$$

It is straightforward to show that $q$ is the inverse of $p$. This final part of the proof uses arguments that are similar to the ones presented after Theorems 2.3 and 3.1 .

From now on, let $H$ be a weak bialgebra. We have seen in Lemmas 1.3 and 1.6 that $H_{t}=\bar{H}_{t}$ and $H_{s}=\bar{H}_{s}$ are subalgebras of $H$. It was shown in [17, Prop. 1.6] that $H_{s}$ and $H_{t}$ are in fact separable Frobenius algebras.

Proposition 4.4. Let $H$ be a weak bialgebra. Then $H_{t}$ and $H_{s}$ are Frobenius separable $k$-algebras. The separability idempotents of $H_{t}$ and $H_{s}$ are

$e_{t}=\varepsilon_{t}\left(1_{(1)}\right) \otimes 1_{(2)}=1_{(2)} \otimes \bar{\varepsilon}_{t}\left(1_{(1)}\right)$ and $e_{s}=1_{(1)} \otimes \varepsilon_{s}\left(1_{(2)}\right)=\bar{\varepsilon}_{s}\left(1_{(2)}\right) \otimes 1_{(1)}$. The Frobenius systems for $H_{t}$ and $H_{s}$ are respectively $\left(e_{t}, \varepsilon_{\mid H_{t}}\right)$ and $\left(e_{s}, \varepsilon_{\mid H_{s}}\right)$.

Proof. We include a proof for the sake of completeness. Let $e_{t}=\varepsilon_{t}\left(1_{(1)}\right) \otimes$ $1_{(2)}=e^{<1>} \otimes e^{<2>}$. For all $z \in H_{t}$, we have that

$$
\begin{gathered}
\left\langle\varepsilon, z \varepsilon_{t}\left(1_{(1)}\right)\right\rangle 1_{(2)}=\left\langle\varepsilon, z 1_{\left(2^{\prime}\right)}\right\rangle\left\langle\varepsilon, 1_{\left(1^{\prime}\right)} 1_{(1)}\right\rangle 1_{(2)} \\
\stackrel{(r m)}{=}\left\langle\varepsilon, z 1_{(1)}\right\rangle 1_{(2)}=\bar{\varepsilon}_{t}(z)=z ; \\
\left.\varepsilon_{t}\left(1_{(1)}\right) \underset{\left({ }^{\prime m}\right)}{=} 1_{(2)} z\right\rangle=\left\langle\varepsilon, 1_{\left(1^{\prime}\right)} 1_{(1)}\right\rangle\left\langle\varepsilon, 1_{(2)} z\right\rangle 1_{\left(2^{\prime}\right)} \\
\stackrel{(\ln }{=}\left\langle\varepsilon, 1_{\left(1^{\prime}\right)} z\right\rangle 1_{\left(2^{\prime}\right)}=\varepsilon_{t}(z)=z .
\end{gathered}
$$

Taking $z=1$ in (30, 31), we find that $\varepsilon\left(e^{<1>}\right) e^{<2>}=e^{<1>} \varepsilon\left(e^{<2>}\right)=1$.

Then we compute for all $z \in H_{t}$ that

$$
z e^{<1>} \otimes e^{<2>}=z \varepsilon_{t}\left(1_{(1)}\right) \otimes 1_{(2)} \stackrel{(*)}{=} \varepsilon_{t}\left(1_{\left(1^{\prime}\right)}\right)\left\langle\varepsilon, 1_{\left(2^{\prime}\right)} z \varepsilon_{t}\left(1_{(1)}\right)\right\rangle \otimes 1_{(2)}
$$




$$
\stackrel{(* *)}{=} \varepsilon_{t}\left(1_{\left(1^{\prime}\right)}\right) \otimes 1_{\left(2^{\prime}\right)} z=e^{<1>} \otimes e^{<2>} z .
$$

At $(*)$, we applied (31) with $z$ replaced by $z \varepsilon_{t}\left(1_{(1)}\right)$, and at $(* *)$, we applied (30) with $z$ replaced by $1_{\left(2^{\prime}\right)} z$. Finally, it is obvious that $e^{<1>} e^{<2>}=$ $\varepsilon_{t}\left(1_{(1)}\right) 1_{(2)}=1$.

It follows from Propositions 4.1 and 4.4 that $H_{s}$ and $H_{t}$ are coseparable Frobenius coalgebras. The comultiplication is given by

$$
\Delta_{t}(z)=z \varepsilon_{t}\left(1_{(1)}\right) \otimes 1_{(2)}=\varepsilon_{t}\left(z 1_{(1)}\right) \otimes 1_{(2)} \stackrel{22, \text {, 117] }}{=} \varepsilon_{t}\left(1_{(1)} z\right) \otimes \varepsilon_{t}\left(1_{(2)}\right)
$$

(where the second equality follows by Lemma 1.6), so that the comultiplication coincides with the comultiplication introduced in Proposition 1.17.

For $M, N \in{ }_{H} \mathcal{M}$, in terms of the $H_{t}$-actions obtained in the proof of Theorem 2.4, we compute the projection $\pi: M \otimes N \rightarrow M \otimes N$ from Proposition 4.3 .

$$
\pi(m \otimes n)=m \triangleleft \varepsilon_{t}\left(1_{(1)}\right) \otimes 1_{(2)} \triangleright n=\left(\bar{\varepsilon}_{s} \circ \varepsilon_{t}\right)\left(1_{(1)}\right) m \otimes 1_{(2)} n=1 \cdot(m \otimes n) .
$$

Thus $\pi$ coincides with the map $\pi$ introduced after Theorem 2.3 . From Proposition 4.3, we now have an alternative derivation of the following result, which follows also by Theorem 2.4.

Corollary 4.5. Let $H$ be a weak bialgebra, and $M, N \in{ }_{H} \mathcal{M}$. Then $M$ and $N$ are $H_{t}$-bimodules and $H_{t}$-bicomodules, and

$$
M \otimes^{H_{t}} N=M \otimes^{l} N \cong M \otimes_{l} N=M \otimes_{H_{t}} N .
$$

For $M, N \in \mathcal{M}^{H}$, we compute in terms of the $H_{s}$-actions obtained in the proof of Theorem 3.2 the projection $\pi: M \otimes N \rightarrow M \otimes N$ from Proposition 4.3; for $m \in M, n \in N$, we find

$$
\begin{aligned}
\pi(m \otimes n) & =m \triangleleft 1_{(1)} \otimes \varepsilon_{s}\left(1_{(2)}\right) \triangleright n \\
& =\left\langle\varepsilon, m_{[1]} 1_{(1)}\right\rangle\left\langle\varepsilon, 1_{\left(1^{\prime}\right)} n_{[1]}\right\rangle\left\langle\varepsilon, 1_{(2)} 1_{\left(2^{\prime}\right)}\right\rangle m_{[0]} \otimes n_{[0]} \\
& =\left\langle\varepsilon, m_{[1]} n_{[1]}\right\rangle m_{[0]} \otimes n_{[0]}=\varepsilon \cdot(m \otimes n) .
\end{aligned}
$$

We conclude that $\pi$ coincides with the map $\pi$ introduced after Theorem 3.1 . From Proposition 4.3, we now have an alternative derivation of the following result, which follows also by Theorem 3.2 .

Corollary 4.6. Let $H$ be a weak bialgebra, and $M, N \in \mathcal{M}^{H}$. Then $M$ and $N$ are $H_{s}$-bimodules and $H_{s}$-bicomodules, and

$$
M \otimes^{H_{s}} N=M \otimes^{r} N \cong M \otimes_{r} N=M \otimes_{H_{s}} N .
$$

\section{REFERENCES}

[1] G. Böhm, Hopf algebroids, in "Handbook of Algebra" 6, Elsevier, 2009, 173-235.

[2] G. Böhm, S. Lack and R.H. Street, Weak bimonads and weak Hopf monads, J. Algebra 328 (2011), 1-30.

[3] G. Böhm, F. Nill, K. Szlachányi, Weak Hopf algebras I. Integral theory and $C^{*}$ structure, J. Algebra 221 (1999), 385-438. 
[4] G. Böhm, K. Szlachányi, Weak Hopf algebras II. Representation theory, dimensions, and the Markov Trace, J. Algebra 233 (2000), 156-212.

[5] T. Brzeziński, Towers of corings, Comm. Algebra (2003), 2015-2026.

[6] T. Brzeziński, Comodules and corings, in "Handbook of Algebra vol. 6" (ed. M. Hazewinkel), Elsevier, 2009, pp. 237-318.

[7] S. Caenepeel, B. Ion, G. Militaru, The structure of Frobenius algebras and separable algebras, K-Theory 19 (2000), 365-402.

[8] S. Dăscălescu, C. Năstăsescu and Ş. Raianu, "Hopf algebras: an Introduction", Monographs Textbooks in Pure Appl. Math. 235 Marcel Dekker, New York, 2001.

[9] C. Kassel, "Quantum Groups", Graduate Texts Math. 155, Springer Verlag, Berlin, 1995.

[10] R. G. Larson, Coseparable Hopf algebras, J. Pure Appl. Algebra 3 (1973), 261-267.

[11] S. Montgomery, "Hopf algebras and their actions on rings", American Mathematical Society, Providence, 1993.

[12] D. Nikshych, L. Vainerman, Finite quantum groupoids and their applications, in "New directions in Hopf algebras", Math. Sci. Res. Inst. Publ. 43, Cambridge University Press, Cambridge, 2002, 211-262.

[13] F. Nill, Axioms for weak bialgebras, preprint q-alg/9805104.

[14] C. Pastro, R. Street, Weak Hopf monoids in braided monoidal categories, Algebra Number Theory 3 (2009), 149-207.

[15] P. Schauenburg, Weak Hopf algebras and quantum groups, Banach Center Publ. 61, Polish Acad. Sci., Warsaw, 2003, 171-188.

[16] R. Street, Frobenius monads and pseudomonoids, J. Math. Phys. 45 (2004), 39303948.

[17] K. Szlachányi, Finite quantum groupoids and inclusions of finite type, Fields Inst. Comm. 30, Amer. Math. Soc., Providence, 2001, 393-407.

[18] K. Szlachányi, Adjointable monoidal functors and quantum groupoids. [in:] "Hopf algebras in noncommutative geometry and physics", Lecture Notes in Pure and Appl. Math., 239, Dekker, New York, 2005, 291-307.

Research Institute for Particle and nuclear Physics, P.O.B. 49, H-1525 Budapest 114, Hungary

E-mail address: g.bohm@rmki.kfki.hu

$U R L:$ http://www.rmki.kfki.hu/ ${ }^{\text {bgabr/ }}$

Faculty of Engineering, Vrije Universiteit Brussel, B-1050 Brussels, Belgium

E-mail address: scaenepe@vub.ac.be

$U R L:$ http://homepages.vub.ac.be/ ${ }^{\sim}$ scaenepe/

Faculty of Engineering, Vrije Universiteit Brussel, B-1050 Brussels, Belgium E-mail address: krjanssen@hotmail.com

$U R L:$ http://homepages.vub.ac.be/ krjansse/ 